\title{
Renormalized second-order perturbation theory for the electron correlation energy: Concept, implementation, and benchmarks
}

\author{
Xinguo Ren, ${ }^{1,2}$ Patrick Rinke, ${ }^{1}$ Gustavo E. Scuseria, ${ }^{3}$ and Matthias Scheffler ${ }^{1}$ \\ ${ }^{1}$ Fritz-Haber-Institut der Max-Planck-Gesellschaft, Faradayweg 4-6, 14195, Berlin, Germany \\ ${ }^{2}$ Key Laboratory of Quantum Information, University of Science and Technology of China, Hefei, Anhui 230026, China \\ ${ }^{3}$ Department of Chemistry and Department of Physics \& Astronomy, Rice University, Houston, Texas 77005, USA
}

(Received 14 December 2012; revised manuscript received 28 May 2013; published 17 July 2013)

\begin{abstract}
We present a renormalized second-order perturbation theory (rPT2), based on a Kohn-Sham (KS) reference state, for the electron correlation energy that includes the random-phase approximation (RPA), second-order screened exchange (SOSEX), and renormalized single excitations (rSE). These three terms all involve a summation of certain types of diagrams to infinite order, and can be viewed as "renormalization" of the second-order direct, exchange, and single-excitation (SE) terms of Rayleigh-Schrödinger perturbation theory based on a KS reference. In this work, we establish the concept of rPT2 and present the numerical details of our SOSEX and rSE implementations. A preliminary version of rPT2, in which the renormalized SE (rSE) contribution was treated approximately, has already been benchmarked for molecular atomization energies and chemical reaction barrier heights and shows a well-balanced performance [J. Paier et al., New J. Phys. 14, 043002 (2012)]. In this work, we present a refined version of rPT2, in which we evaluate the rSE series of diagrams rigorously. We then extend the benchmark studies to noncovalent interactions, including the rare-gas dimers, and the S22 and S66 test sets, as well as the cohesive energy of small copper clusters, and the equilibrium geometry of 10 diatomic molecules. Despite some remaining shortcomings, we conclude that rPT2 gives an overall satisfactory performance across different electronic situations, and is a promising step towards a generally applicable electronic-structure approach.
\end{abstract}

DOI: 10.1103/PhysRevB.88.035120

\section{INTRODUCTION}

Density-functional theory ${ }^{1,2}$ (DFT) has played a significant role in first-principles electronic-structure calculations in physics, chemistry, materials science, and biophysics over the past decades. DFT offers an in principle exact formalism for computing ground-state energies of electronic systems, but in practice the exchange-correlation (XC) energy functional has to be approximated. Existing approximations to the XC functional can be classified into different rungs according to a hierarchical scheme known as "Jacob's ladder." 3 The random-phase approximation (RPA), ${ }^{4,5}$ which in the context of DFT (Refs. 6 and 7) amounts to treating the exchange energy exactly and the correlation energy at the level of RPA, is on the fifth and highest rung of this ladder. RPA has received considerable attention (for recent reviews, see Refs. 8-10) since its first application to realistic systems. ${ }^{11}$ This is largely due to the fact that RPA has shown great promise in resolving difficulties encountered by the local-density and generalized gradient approximations (LDA/GGAs) to DFT. The resolution of the "CO adsorption puzzle,"12-14 the encouraging behavior for the "strongly correlated" $f$-electron metal cerium,,${ }^{15}$ and the excellent performance of RPA (and its variants) across a wide range of systems including solids, ${ }^{14,16,17}$ van der Waals (vdW) bonded molecules, ${ }^{18-22}$ and thermochemistry ${ }^{23}$ are just a few examples.

Quantitatively, however, RPA itself does not always provide the desired accuracy. It was found empirically that the common practice of evaluating both the exact-exchange and the RPA correlation energy in a post-processing way using Kohn-Sham (KS) or generalized KS orbitals leads to a systematic underestimation of bond strengths in both molecules and solids. ${ }^{11,14,21,24}$ Iterating RPA to self-consistency does not alleviate this problem. ${ }^{25}$ Various attempts have been made in the past to
PACS number(s): 71.15.Mb, 31.15.ae, 31.15.vq, 31.15.xp

improve the standard RPA scheme, ${ }^{18,20,21,24,26-32}$ with varying degrees of success. Here, we will focus on two flavors of beyond-RPA schemes that both alleviate the underbinding problem of RPA: the second-order screened exchange ${ }^{24,27,33}$ (SOSEX) and the single-excitation (SE) correction. ${ }^{21}$ SOSEX was originally formulated in the context of coupled-cluster theory, ${ }^{27,33}$ and accounts for the antisymmetric nature of the many-electron wave function. Like RPA, it can be interpreted as an infinite summation of a set of topologically similar diagrams. ${ }^{10,27,34}$ Adding SOSEX to RPA makes the theory oneelectron "self-correlation" free. The SE correction, on the other hand, accounts for the fact that the KS orbitals are not optimal for a post-processing perturbation treatment at the exactexchange level. ${ }^{21}$ Inspired by the diagrammatic representation of RPA and SOSEX, one can also identify a sequence of topologically similar diagrams of single-excitation character. Summing these to infinite order yields what we called the renormalized single-excitation (rSE) contribution ${ }^{21}$ to the electron correlation energy. Combining all three contributions, RPA, SOSEX, and rSE, leads to the "RPA + SOSEX + rSE" scheme or, as we shall refer to it in this work, renormalized secondorder perturbation theory, in short rPT2 (note that in Ref. 10 we used the acronym $\mathrm{r} 2 \mathrm{PT}$ ). The name is inspired by secondorder Rayleigh-Schrödinger perturbation theory (RSPT) that becomes renormalized through the infinite summations. This can be compared to the commonly used second-order MøllerPlesset (MP2) method, which is the straight (bare) secondorder RSPT based on the Hartree-Fock reference.

A preliminary version of $\mathrm{rPT} 2$, in which an approximate treatment of rSE was invoked, had been benchmarked for atomization energies of molecules and chemical reaction barrier heights in Ref. 35 . We found that rPT2 gives the "most balanced" performance compared to other RPA-based 
schemes. However, this approximate treatment of rSE turns out to be problematic for weak interactions and exhibits an unphysical behavior in, e.g., the binding-energy curve of rare-gas dimers. In this work, we will show how a rigorous evaluation of rSE can be carried out. From here on, rPT2 will refer to this revised scheme and not the approximate version presented in Ref. 35. We will, in particular, examine the performance of rPT2 for weakly bonded molecules, including rare-gas dimers, and the widely used S22 and S66 test sets of Hobza and coauthors. Additionally, we will present the cohesive energies of selected small copper clusters as well as the the equilibrium bond lengths of a set of diatomic molecules. ${ }^{36-38}$ For completeness, we will also revisit the benchmark sets for the $\mathrm{G} 2$ atomization energies of Curtiss et al. ${ }^{39}$ and the chemical reaction barrier heights of Truhlar and coauthors ${ }^{40,41}$ for which the performance of the preliminary rPT2 version was first tested in Ref. 35. In addition to the concept of rPT2 and benchmark studies, we will also present a different way of formulating the SOSEX term, which corresponds to the adiabatic connection formulation of SOSEX (AC-SOSEX) by Jansen, Liu, and Ánygán, ${ }^{42}$ and which reflects our actual implementation. Our benchmark studies show that rPT2 represents an overall improvement over RPA, and gives a gratifying performance across different electronic and chemical environments. We also identify remaining shortcomings that will guide further developments of the theory.

The remainder of the paper is organized as follows: In Sec. II, the basic theory and implementation of rPT2 are presented. This is followed by a systematic benchmark test for rPT2 for a range of systems in Sec. III. Conclusions are drawn in Sec. IV. Further details of our implementation and derivations will be given in Appendixes.

\section{THEORY}

In this section, the theoretical foundation of $\mathrm{rPT} 2$ will be presented. We first recapitulate the basics of the RPA + SOSEX method in Sec. II A, and formulate the method in a way that reflects its implementation in the Fritz Haber Institute $a b$ initio molecular simulations (FHI-AIMS) code package. ${ }^{43,44}$ This is followed by the derivation of an algebraic expression for the rSE term, the third ingredient in rPT2. A discussion of the underlying physics behind the rPT2 method is then presented from a diagrammatic point of view in Sec. II C. From the diagrammatic representation it will become clear that the three terms in rPT2 appear at the same level of approximation and hence should be treated at an equal footing.

\section{A. RPA + SOSEX method}

The RPA method can be formulated in different ways (for a review, see Refs. 9 and 10). In the DFT context, RPA can be derived from the adiabatic-connection fluctuation-dissipation (ACFD) theorem, ${ }^{6,7}$ whereby the RPA correlation energy is expressed as

$$
\begin{aligned}
E_{\mathrm{c}}^{\mathrm{RPA}}= & -\frac{1}{2 \pi} \int_{0}^{1} d \lambda \int_{0}^{\infty} d \omega \iint d \boldsymbol{r} d \boldsymbol{r}^{\prime} v\left(\boldsymbol{r}, \boldsymbol{r}^{\prime}\right) \\
& \times\left[\chi_{\lambda}^{\mathrm{RPA}}\left(\boldsymbol{r}^{\prime}, \boldsymbol{r}, i \omega\right)-\chi_{0}\left(\boldsymbol{r}^{\prime}, \boldsymbol{r}, i \omega\right)\right] .
\end{aligned}
$$

$\chi_{0}(i \omega)$ is the $\mathrm{KS}$ independent-particle density-response function

$$
\chi_{0}\left(\boldsymbol{r}, \boldsymbol{r}^{\prime}, i \omega\right)=\sum_{i a}\left[\frac{\psi_{i}^{*}(\mathbf{r}) \psi_{\mathbf{a}}(\mathbf{r}) \psi_{\mathbf{i}}\left(\mathbf{r}^{\prime}\right) \psi_{\mathbf{a}}\left(\mathbf{r}^{\prime}\right)}{\epsilon_{i}-\epsilon_{a}-i \omega}+\text { c.c. }\right]
$$

where $\psi_{i, a}(\mathbf{r})$ and $\epsilon_{i, a}$ are the KS single-particle orbitals and orbital energies, and c.c. the "complex conjugate." Here and in the following, we adopt the following convention: $i, j$ correspond to occupied and $a, b$ to unoccupied (or virtual) spin orbitals, whereas $p, q$ apply to general cases. $\chi_{\lambda}^{\mathrm{RPA}}(i \omega)$ in Eq. (1) is the RPA response function of a fictitious system with a scaled Coulomb interaction $\frac{\lambda}{\left|\mathbf{r}-\mathbf{r}^{\prime}\right|}$ (with $0 \leqslant \lambda \leqslant 1$, and satisfies the Dyson equation

$$
\chi_{\lambda}^{\mathrm{RPA}}=\chi_{0}+\chi_{0} \lambda v \chi_{\lambda}^{\mathrm{RPA}} .
$$

Representing $\chi_{0}$ and $v$ in the "particle-hole basis" $\left\{\psi_{i}^{*}(\mathbf{r}) \psi_{\mathbf{a}}(\mathbf{r}), \psi_{\mathbf{a}}^{*}(\mathbf{r}) \psi_{\mathbf{i}}(\mathbf{r})\right\}$, one can obtain the RPA correlation energy by solving the following eigenvalue problem $^{11}$ :

$$
\left(\begin{array}{rr}
A & B^{*} \\
-B & -A^{*}
\end{array}\right)\left(\begin{array}{c}
X_{n} \\
Y_{n}
\end{array}\right)=\left(\begin{array}{c}
X_{n} \\
Y_{n}
\end{array}\right) \omega_{n}
$$

where $A_{i a, j b}=\left(\epsilon_{a}-\epsilon_{i}\right) \delta_{i j} \delta_{a b}+\langle i b \mid a j\rangle, \quad$ and $\quad B_{i a, j b}=$ $\langle i j \mid a b\rangle$. The two-electron Coulomb integrals are

$$
\langle p q \mid r s\rangle=\iint d x_{1} d x_{2} \frac{\psi_{p}^{*}\left(x_{1}\right) \psi_{r}\left(x_{1}\right) \psi_{q}^{*}\left(x_{2}\right) \psi_{s}\left(x_{2}\right)}{\left|\mathbf{r}_{1}-\mathbf{r}_{2}\right|},
$$

where $x=(\mathbf{r}, \sigma)$ is a combined space-spin variable. As demonstrated by Furche, ${ }^{45}$ after solving Eq. (4), the RPA correlation energy can be written as

$$
E_{\mathrm{c}}^{\mathrm{RPA}}=\frac{1}{2} \operatorname{Tr}(\omega-A)=\frac{1}{2}\left[\sum_{n}^{\prime} \omega_{n}-\sum_{i a} A_{i a, i a}\right],
$$

where $\sum_{n}^{\prime}$ implies that the summation over $n$ is restricted to positive eigenvalues $\omega_{n}$.

Scuseria et al. demonstrated that an equivalent formulation of the RPA correlation energy of Eq. (6) can be obtained from an approximate coupled-cluster doubles (CCD) theory ${ }^{46}$ in which only the "ring diagrams" are kept (see the first row of Fig. 1). In the CCD theory, only double excitation contributions are included in the "cluster operator" which generates the interacting many-body ground-state wave function through the exponential ansatz. By contrast, in the more often used coupled-cluster singles-doubles (CCSD) approach, both single and double excitations are included. Within the CCD formulation of RPA, the key quantities are the (direct) ring-CCD amplitudes $T_{i a, j b}$, which (in the case of real canonical spin orbitals) are determined by the following Riccati equation:

$$
B+A T+T A+T B T=0 .
$$

Due to the quadratic nature of this equation, one should take care to ensure that the physical solution is taken. ${ }^{47}$ The RPA correlation energy in this ring-CCD formulation is then given by

$$
E_{\mathrm{c}}^{\mathrm{RPA}}=\frac{1}{2} \operatorname{Tr}(B T)=\frac{1}{2} \sum_{i j, a b}\langle i j \mid a b\rangle T_{j b, i a} .
$$




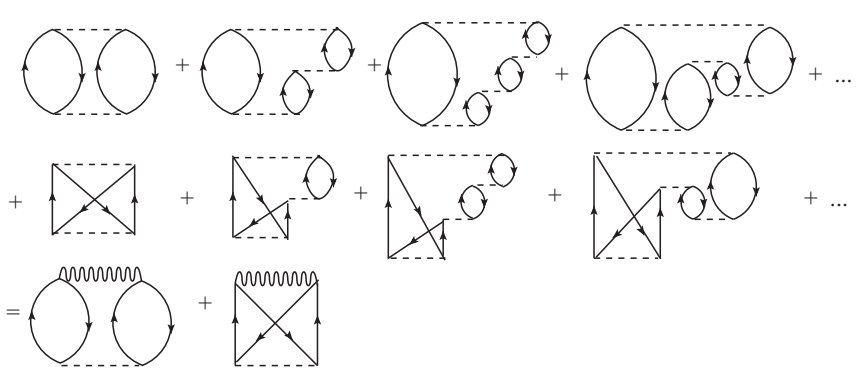

FIG. 1. Goldstone diagrams for RPA (first row) and SOSEX (second row) contributions. Dashed lines represent bare Coulomb interactions, and full lines correspond to KS electrons (arrow up) and holes (arrow down). Third row: RPA + SOSEX energy in the coupled-cluster context. The wiggly together with the arrowed solid lines represent the direct ring-CCD amplitudes $T_{i a, j b}$ [see Eq. (9)]. The contraction between the direct ring-CCD amplitudes and the bare Coulomb interactions (dashed lines) yields the RPA + SOSEX correlation energy.

We note that this is often called direct RPA in the quantum chemistry literature to emphasize the fact that higher-order exchange-type contributions are not included.

Now, the RPA + SOSEX correlation energy can be conveniently introduced ${ }^{24,27,33}$ by antisymmetrizing the Coulomb integral in Eq. (8), ${ }^{27}$

$$
E_{\mathrm{c}}^{\mathrm{RPA}+\mathrm{SOSEX}}=\frac{1}{2} \operatorname{Tr}(\tilde{B} T)=\frac{1}{2} \sum_{i j, a b}\langle i j \| a b\rangle T_{j b, i a},
$$

where $\tilde{B}_{i a, j b}=\langle i j|| a b\rangle=\langle i j \mid a b\rangle-\langle i j \mid b a\rangle$. The SOSEX correction term itself is

$$
E_{\mathrm{c}}^{\mathrm{SOSEX}}=-\frac{1}{2} \sum_{i j, a b}\langle i j \mid b a\rangle T_{j b, i a} .
$$

Physically, the SOSEX correction introduces higher-order exchange processes that can also be represented by an infinite summation of Goldstone diagrams (see the second row of Fig. 1). This infinite summation is condensed into the ring-CCD amplitudes whose contraction with the bare Coulomb interaction (after antisymmetrization) yields the RPA + SOSEX correlation energy as illustrated by the thirdrow diagrams in Fig. 1.
In a coupled-cluster code, the SOSEX energy can be readily computed once the direct ring-CCD amplitudes $T_{i a, j b}$ are available. A slightly different variant of SOSEX can be obtained in the ACFD framework, as shown in Ref. 42. We will show later that, although not identical, these two SOSEX formulations produce very similar results. Our implementation in the FHI-AIMS code ${ }^{43,44}$ follows the ACFD route. To illustrate our approach, let us first present an alternative way to Eq. (1) of expressing the RPA correlation energy within ACFD before we introduce the corresponding SOSEX extension. Equation (3) yields

$$
\begin{aligned}
\chi_{\lambda}^{\mathrm{RPA}}(i \omega)= & \chi_{0}(i \omega)+\chi_{0}(i \omega) \lambda v \chi_{\lambda}^{\mathrm{RPA}}(i \omega) \\
= & \chi_{0}(i \omega)+\lambda \chi_{0}(i \omega) v \chi_{0}(i \omega) \\
& +\lambda^{2} \chi_{0}(i \omega) v \chi_{0}(i \omega) v \chi_{0}(i \omega)+\cdots .
\end{aligned}
$$

The RPA correlation energy in Eq. (1) can then be rewritten as

$$
\begin{aligned}
E_{\mathrm{c}}^{\mathrm{RPA}}= & -\frac{1}{2 \pi} \int_{0}^{1} d \lambda \int_{0}^{\infty} d \omega \operatorname{Tr}\left[\chi_{0}(i \omega) v \chi_{0}(i \omega) \cdot \lambda v\right. \\
& \left.+\chi_{0}(i \omega) v \chi_{0}(i \omega) \cdot \lambda^{2} v \chi_{0}(i \omega) v+\cdots\right] \\
= & -\frac{1}{2 \pi} \int_{0}^{1} d \lambda \int_{0}^{\infty} d \omega \operatorname{Tr}\left[\chi_{0}(i \omega) v \chi_{0}(i \omega) W_{\lambda}(i \omega)\right] \\
= & -\frac{1}{2 \pi} \int_{0}^{\infty} d \omega \operatorname{Tr}\left[\chi_{0}(i \omega) v \chi_{0}(i \omega) \bar{W}(i \omega)\right]
\end{aligned}
$$

where

$$
W_{\lambda}(i \omega)=\lambda v /\left[1-\lambda \chi_{0}(i \omega) v\right]
$$

is the coupling-constant-dependent screened Coulomb interaction and

$$
\bar{W}(i \omega)=\int_{0}^{1} d \lambda W_{\lambda}(i \omega)
$$

the coupling-constant-averaged screened Coulomb interaction. In this context, we would like to point out that the first diagram in the third row of Fig. 1 can alternatively be interpreted as the pictorial representation of Eq. (13). Now, the bubbles correspond to $\chi_{0}$, dashed lines to the bare Coulomb interaction, and wiggly lines to the corresponding screened interaction $\bar{W}(i \omega)$.

Expressing $\chi_{0}$ again in terms of the "particle-hole basis" [defined below Eq. (3)] and using Eq. (2), Eq. (13) can be recast into

$$
\begin{aligned}
E_{c}^{\mathrm{RPA}}= & \frac{1}{2 \pi} \int_{0}^{\infty} d \omega \sum_{i a, j b}\left[\frac{\langle a j \mid i b\rangle\langle i b|\bar{W}(i \omega)| a j\rangle}{\left(\epsilon_{i}-\epsilon_{a}-i \omega\right)\left(\epsilon_{j}-\epsilon_{b}-i \omega\right)}\right. \\
& \left.+\frac{\langle a b \mid i j\rangle\langle i j|\bar{W}(i \omega)| a b\rangle}{\left(\epsilon_{i}-\epsilon_{a}-i \omega\right)\left(\epsilon_{j}-\epsilon_{b}+i \omega\right)}+\frac{\langle i j \mid a b\rangle\langle a b|\bar{W}(i \omega)| i j\rangle}{\left(\epsilon_{i}-\epsilon_{a}+i \omega\right)\left(\epsilon_{j}-\epsilon_{b}-i \omega\right)}+\frac{\langle i b \mid a j\rangle\langle a j|\bar{W}(i \omega)| i b\rangle}{\left(\epsilon_{i}-\epsilon_{a}+i \omega\right)\left(\epsilon_{j}-\epsilon_{b}+i \omega\right)}\right],
\end{aligned}
$$

where $\langle p q|\bar{W}(i \omega)| r s\rangle$ is defined in analogy to $\langle p q \| r s\rangle$ in Eq. (5), by replacing the bare Coulomb interaction $v$ by the screened (and frequency-dependent) one $\bar{W}(i \omega)$.

For real canonical spin orbitals, one has $\langle a j \mid i b\rangle=$ $\langle i b \mid a j\rangle=\langle a b \mid i j\rangle=\langle i j \mid a b\rangle$. The same relations hold for the screened Coulomb repulsion integrals. The above equation then simplifies to

$$
\begin{aligned}
E_{c}^{\mathrm{RPA}}= & \frac{1}{2 \pi} \int_{0}^{\infty} d \omega \sum_{i a, j b}\langle i j \mid a b\rangle\langle i j|\bar{W}(i \omega)| a b\rangle \\
& \times \mathcal{F}_{i a}(i \omega) \mathcal{F}_{j b}(i \omega)
\end{aligned}
$$


with the factors

$$
\mathcal{F}_{i a}(i \omega)=2\left(\epsilon_{i}-\epsilon_{a}\right) /\left[\left(\epsilon_{i}-\epsilon_{a}\right)^{2}+\omega^{2}\right] .
$$

Now, in analogy to the (direct) ring-CCD formulation of SOSEX in Eq. (10), one can obtain a corresponding SOSEX term (the so-called "AC-SOSEX") from Eq. (17), by exchanging the " $a, b$ " indices in $\langle i j \mid b a\rangle$ (with an additional minus sign)

$$
\begin{aligned}
E_{c}^{\mathrm{AC}-S O S E X}= & -\frac{1}{2 \pi} \int_{0}^{\infty} d \omega \sum_{i a, j b}\langle i j \mid b a\rangle\langle i j|\bar{W}(i \omega)| a b\rangle \\
& \times \mathcal{F}_{i a}(i \omega) \mathcal{F}_{j b}(i \omega) .
\end{aligned}
$$

Then, using the resolution-of-identity technique, ${ }^{44,48-50}$ Eq. (19) can be implemented with relative ease. The implementation details of Eq. (19) in FHI-AIMS are presented in Appendix A.

To make closer contact with the expression given in Ref. 42, we note that Eq. (17) can be further rewritten as

$$
E_{c}^{\mathrm{AC}-\operatorname{SOSEX}}=-\frac{1}{2} \sum_{i a, j b}\langle i j \mid b a\rangle \bar{P}_{i a, j b},
$$

where

$$
\bar{P}_{i a, j b}=\frac{1}{\pi} \int_{0}^{\infty} d \omega\langle i j|\bar{W}(i \omega)| a b\rangle \mathcal{F}_{i a}(i \omega) \mathcal{F}_{j b}(i \omega)
$$

is the coupling-strength-averaged (two-particle) density matrix.

As shown by Jansen, Liu, and Ánygán, ${ }^{42}$ Eq. (20) is usually not identical to the original ring-CCD based SOSEX in Eq. (10) (except for one- and two-electron cases). However, the difference between them is very small (relative difference in RPA + SOSEX correlation energy less that $0.15 \%$ for atoms and small molecules), as first noted in Ref. 31 and also confirmed here. In Appendix B, the RPA and (AC-)SOSEX correlation energies, as well as the corresponding atomization energies, are presented for five small molecules.

Our benchmark results presented in Sec. III are based on the AC-SOSEX scheme. However, since the numerical difference between the two SOSEX flavors is insignificant, at least for small molecules, we expect that our conclusion should also apply to the original ring-CCD based SOSEX as well.

\section{B. rSE correction and the semicanonicalization method}

In Ref. 21, we showed that the correlation energy arising from single excitations is an important term, and adding this to the RPA correlation energy improves the molecular binding energies considerably. The single-excitation correction derives directly from Rayleigh-Schrödinger perturbation theory (RSPT) and adopts a simple form in terms of the single-particle orbitals

$$
E_{\mathrm{c}}^{\mathrm{SE}}=\sum_{i a} \frac{\left|\left\langle\psi_{i}|\hat{f}| \psi_{a}\right\rangle\right|^{2}}{\epsilon_{i}-\epsilon_{a}}=\sum_{i a} \frac{\left|f_{i a}\right|^{2}}{\epsilon_{i}-\epsilon_{a}} .
$$

Here, $\left|\psi_{i(a)}\right\rangle$ and $\epsilon_{i(a)}$ refer to occupied (unoccupied) KohnSham (KS) orbitals and the corresponding orbital energies. $\hat{f}$ is the single-particle Hartree-Fock (HF) Hamiltonian, or the so-called Fock operator. A detailed derivation of Eq. (22) has been given in the Supplemental Material of Ref. 21.
Denoting the single-particle KS Hamiltonian $\hat{h}^{0}$, we obtain $\left\langle\psi_{i}|\hat{f}| \psi_{a}\right\rangle=\left\langle\psi_{i}\left|\hat{h}^{0}+\Delta \hat{v}\right| \psi_{a}\right\rangle=\left\langle\psi_{i}|\Delta \hat{v}| \psi_{a}\right\rangle$ when $\psi_{i}, \psi_{a}$ are eigenfunctions of $\hat{h}^{0} . \Delta \hat{v}$ is the difference between the $\mathrm{HF}$ exact-exchange potential and the KS exchange-correlation potential. A similar SE contribution is encountered in the context of KS many-body perturbation theory. ${ }^{51-53}$ However, we emphasize that here we followed the procedure of RSPT to derive Eq. (22), instead of the ACFD formalism, which requires the electron-density to be fixed along the adiabaticconnection path. Whether the two procedures will yield significantly different results is a subject of further studies.

From the viewpoint of RSPT, Eq. (22) represents one of the three contributions to second-order correlation energy. This term suffers from the same divergence problem as secondorder Møller-Plesset perturbation theory for metallic systems when the single-particle KS gap closes. A remedy suggested in Ref. 21 was to follow the RPA spirit and to sum a sequence of higher-order SE terms to infinite order. Such higher-order $\mathrm{SE}$ terms can be represented in terms of Goldstone diagrams, as illustrated in Fig. 2. We refer to this infinite summation of SE terms as renormalized single excitations (rSE) as alluded to in the Introduction.

The influence of the rSE correction was first examined in Ref. 35, albeit in an approximate way. There a so-called "diagonal" approximation to rSE (denoted here as "rSEdiag") was used, in which only terms with " $i=j=k=$ ..." and " $a=b=c=\ldots$." were included. The remaining "off-diagonal" terms were omitted. A similar approximation has been used in summing up the Epstein-Nesbet ladder-type diagrams in Ref. 53. In this way, the sequence of diagrams falls into a geometrical series. Summing them up yields the following simple expression:

$$
E_{\mathrm{c}}^{\mathrm{rSE}-\mathrm{diag}}=\sum_{i a} \frac{\left|f_{i a}\right|^{2}}{\epsilon_{i}-\epsilon_{a}+\Delta v_{i i}-\Delta v_{a a}},
$$

where $\Delta v_{p q}=\left\langle\psi_{p}|\Delta \hat{v}| \psi_{q}\right\rangle$. The additional term $\Delta v_{i i}-\Delta v_{a a}$ that appears in the denominator is negative definite and removes the divergence problem even for vanishing KS gaps. The addition of rSE-diag to RPA and RPA + SOSEX has been benchmarked for atomization energies and reaction barriers in Ref. 35. We found that the renormalization (i.e., going from SE to rSE-diag) has a tendency to slightly reduce atomization energies, but the overall effect is not significant. For chemical reaction barrier heights, on the other hand, the renormalization is crucial for the transition states, that typically have a rather small energy gap.

The diagonal approximation in Eq. (23) is not invariant under unitary transformations in the space of occupied and unoccupied orbitals. More importantly, however, it can lead to an unphysical behavior in the potential-energy surface of weakly interacting systems, as will be shown in Sec. III A1. Recently, we discovered that it is straightforward to include the "off-diagonal" elements as well, and to treat the rSE term rigorously. In Appendix $\mathrm{C}$, we illustrate in detail how the infinite summation of the diagrams depicted in Fig. 2 can be carried out. Here, we only present the key steps that lead to the final expression, and that are needed in practical calculations. 


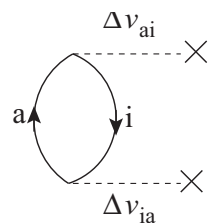

2nd-order

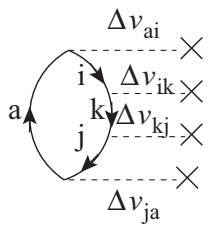

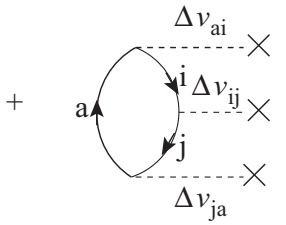

3 rd-order
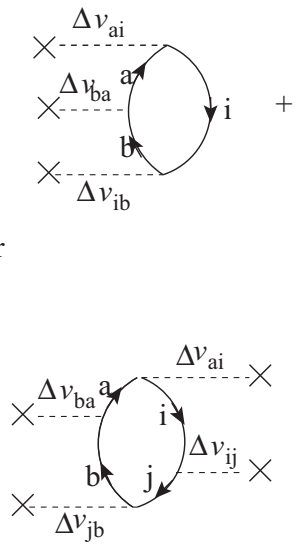

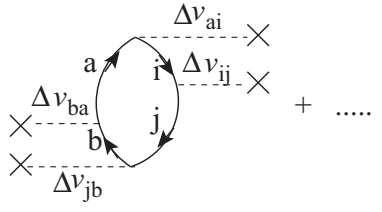

4th-order

FIG. 2. Goldstone diagrams for a sequence of correlation-energy terms arising from single excitations. Summing these up to infinite order yields the renormalized single-excitation (rSE) contribution. Here, $\Delta v_{p q}=\left\langle\psi_{p}\left|\hat{f}-\hat{h}^{0}\right| \psi_{q}\right\rangle$, and note $\Delta v_{i a}=f_{i a}$.

First, the occupied and unoccupied blocks of the Fock matrix (evaluated with KS orbitals) need to be constructed:

$$
\begin{gathered}
f_{i j}=\left\langle\psi_{i}|\hat{f}| \psi_{j}\right\rangle=\epsilon_{i} \delta_{i j}+\Delta v_{i j}, \\
f_{a b}=\left\langle\psi_{a}|\hat{f}| \psi_{b}\right\rangle=\epsilon_{a} \delta_{a b}+\Delta v_{a b} .
\end{gathered}
$$

The second step is to diagonalize the $f_{i j}$ and the $f_{a b}$ blocks separately. Denoting the eigenvector matrices as $\mathcal{O}$ and $\mathcal{U}$, one has

$$
\begin{aligned}
\sum_{k} f_{i k} \mathcal{O}_{k j} & =\mathcal{O}_{i j} \tilde{\epsilon}_{j}, \\
\sum_{c} f_{a c} \mathcal{U}_{c b} & =\mathcal{U}_{a b} \tilde{\epsilon}_{b},
\end{aligned}
$$

where $\tilde{\epsilon}_{j}$ and $\tilde{\epsilon}_{b}$ are the eigenvalues of the occupied and unoccupied blocks of the Fock matrix, respectively. This procedure is known as semicanonicalization in quantum chemistry (see, e.g., Refs. 54 and 55). The final rSE expression, equivalent to the infinite-order diagrammatic summation, is given by

$$
E_{\mathrm{c}}^{\mathrm{rSE}}=\sum_{i a} \frac{\left|\tilde{f}_{i a}\right|^{2}}{\tilde{\epsilon}_{i}-\tilde{\epsilon}_{a}},
$$

where $\tilde{f}_{i a}$ correspond to the "transformed" off-diagonal block of the Fock matrix

$$
\tilde{f}_{i a}=\sum_{j b} \mathcal{O}^{*}{ }_{i j} \mathcal{U}^{*}{ }_{a b} f_{j b} .
$$

This is a surprisingly simple result: The final rSE expression is formally identical to the second-order SE one, only that the meaning of the energy eigenvalues and the transition amplitudes has to be modified. The equivalence of Eq. (25) to the algebraical expression from a direct evaluation of the diagrams in Fig. 2 is demonstrated in Appendix C.

\section{Concept of rPT2 viewed from its diagrammatic representation}

Initially, the RPA + SOSEX and RPA + (r)SE schemes were investigated separately ${ }^{21,27}$ in an effort to improve the accuracy of the RPA method. In Ref. 35 it was found that adding both terms to RPA leads to even better accuracy in general, and that the combined RPA + SOSEX + rSE (三rPT2) scheme represents the most balanced approach for describing both atomization energies and reaction barrier heights. In fact, this approach is theoretically well justified since, as will be shown in the following, all three terms appear at the same level of approximation. To elucidate the nature of rPT2, the Goldstone diagrams for the three ingredients of this theory are shown together in Fig. 3. All three pieces are characterized by an infinite summation of diagrams with the same topological structure. The leading terms in the three series are the second-order direct (Coulomb), the second-order exchange, and the SE term, respectively. In other words, these three leading terms represent exactly secondorder Rayleigh-Schrödinger perturbation theory, based on an (approximate) KS reference Hamiltonian. If the perturbation series were to be built on the HF reference, the SE term would vanish (Brillouin's theorem). ${ }^{56}$ In essence, the theory is exact at second order, and for higher-order contributions we follow the strategy of "selective summation to infinite order," following the spirit of the RPA. This "infinite-order
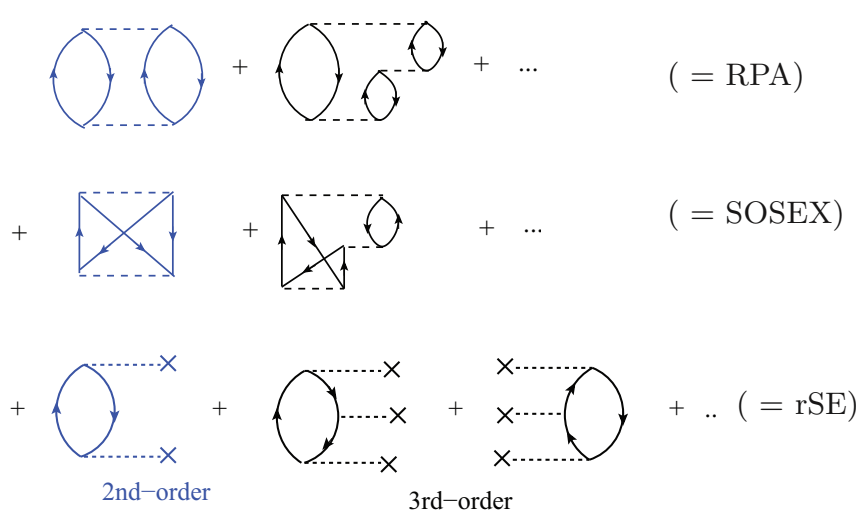

FIG. 3. (Color online) rPT2 represented in terms of Goldstone diagrams. The three rows of (infinitely summed) diagrams represent the three components of rPT2: RPA, SOSEX, and rSE. The first column shows the (only) three terms in normal (bare) second-order Rayleigh-Schrödinger perturbation theory based on a KS reference. 
summation" effectively renormalizes the three terms of the (bare) second-order perturbation theory (PT2), represented by the blue diagrams in Fig. 3. We expect the renormalized method, i.e., rPT2, to be more generally applicable than the bare PT2. The latter works very well for wide-gap molecules, but suffers from notorious divergence problems for systems with zero direct gap. ${ }^{57,58}$

As a perturbation theory, rPT2 will necessarily depend on the reference Hamiltonian or, equivalently, a set of input singleparticle orbitals. In practice, rPT2 works best when based on KS Hamiltonian (i.e., with a local, multiplicative potential) that yield a smaller gap than generalized KS or HF ones. This is directly related to the fact that the underbinding error of RPA will be even more pronounced for HF or generalized KS reference Hamiltonians, as evidenced by the significant RPA@HF error for the $\mathrm{G} 2$ atomization energies, ${ }^{44}$ and the severely underestimated RPA@HF (40\%) $\mathrm{C}_{6}$ coefficients $^{59}$ (here and in the following, we use "method@reference" to denote which method is based on which reference state). For a variety of $\mathrm{KS}$ Hamiltonians, RPA results were found to be insensitive to the actual choice of the reference Hamiltonian. ${ }^{23,60}$ In this work, we will therefore choose the most popular nonempirical GGA functional PBE as the reference, also to be consistent with our previous work..$^{10,21,35}$ The insensitivity of RPA to reference KS Hamiltonians carries over to rPT2.

\section{RESULTS}

In this section, we will benchmark the performance of rPT2 for weak interaction energies (rare-gas dimers, S22 and S66 test sets by Hobza and co-workers ${ }^{36,38}$ ), atomization energies (from the G2-I test set by Curtiss et al. ${ }^{39,61}$ ), and chemical reaction barrier heights (38 hydrogen-transfer and 38 non-hydrogen-transfer chemical reactions of Truhlar and coworkers ${ }^{40,41}$ ). All calculations were performed with the localorbital-based all-electron FHI-AIMS code. ${ }^{43,44}$ As mentioned in Sec. II A, the SOSEX term in this work corresponds to "AC-SOSEX" based on Eq. (19). For brevity, we will simply refer to it as SOSEX in the following. For the frequency integration in our RPA and SOSEX calculations, we use a modified Gauss-Legendre grid ${ }^{44}$ with 40 points. For the $\lambda$ integration in Eq. (15), we use a normal Gauss-Legendre grid with 5 points. These settings guarantee sufficient accuracy for the benchmark studies presented in this work. The basis sets employed in the calculations will be specified later when discussing the results. Convergence tests are shown in Appendix D.

\section{A. Weak interactions}

One prominent feature of RPA-based approaches is that the ubiquitous vdW interactions are captured in a seamless manner. ${ }^{62,63}$ The long-range behavior of the RPA interaction energy between two closed-shell molecular systems decays as $C_{6} / R^{6}$ where the $C_{6}$ value is dictated by the RPA polarizability of the monomer. ${ }^{63,64}$ Many-body terms that go beyond the pair-wise summation are also automatically contained in this approach. $^{65}$

Benchmarking the performance of RPA and related methods for $\mathrm{vdW}$ bonded systems has been a very active enterprise. ${ }^{16,18,20-23,42,66-69}$ It has been demonstrated that the standard RPA approach exhibits a systematic underbinding behavior for molecules, in particular, vdW bonded ones. ${ }^{21} \mathrm{We}$ have previously shown that SE-type corrections ameliorate this problem, ${ }^{21}$ but the influence of the SOSEX correction has not been systematically benchmarked for vdW systems yet, with the exception of $\mathrm{He}_{2}$ and $\mathrm{Ne}_{2} \cdot{ }^{24}$ It is therefore interesting and timely to examine how rPT2, which combines both types of corrections, performs for noncovalent interactions. Some rPT2 results for $\mathrm{Ar}_{2}$ and $\mathrm{S} 22$ have been featured in our recent review on RPA. ${ }^{10}$ Here, we extend the benchmark study to other rare-gas dimers and also the larger S66 test set.

\section{Rare-gas dimers}

First, we demonstrate the pathological behavior of rSE-diag (our previous, approximate version) for weak interactions, highlighting the importance of including the "off-diagonal" terms in the rSE summation to make the theory invariant with respect to orbital rotations. In Fig. 4, the binding energy of $\mathrm{Ar}_{2}$ is plotted for PBE, RPA, and RPA plus different versions of single-excitation corrections (RPA + SE, RPA + rSE-diag, $\mathrm{RPA}+\mathrm{rSE}$ ). While PBE, RPA, and RPA + SE all show their characteristic behaviors, the behavior of RPA + rSEdiag is weird. The binding-energy curve develops unphysical undulations away from equilibrium. Moreover, the asymptotic limit does not follow the correct $1 / R^{6}$ behavior, and the curve even reaches above the energy zero at large bonding distances (see the inset of Fig. 4). It is reassuring, however, to observe that this pathological behavior disappears in the upgraded $\mathrm{RPA}+\mathrm{rSE}$ scheme, which yields a binding energy curve in close agreement with the Tang-Toennies reference curve, ${ }^{70}$ obtained from a simple analytical model with experimental equilibrium bond distance and binding energy as input param-

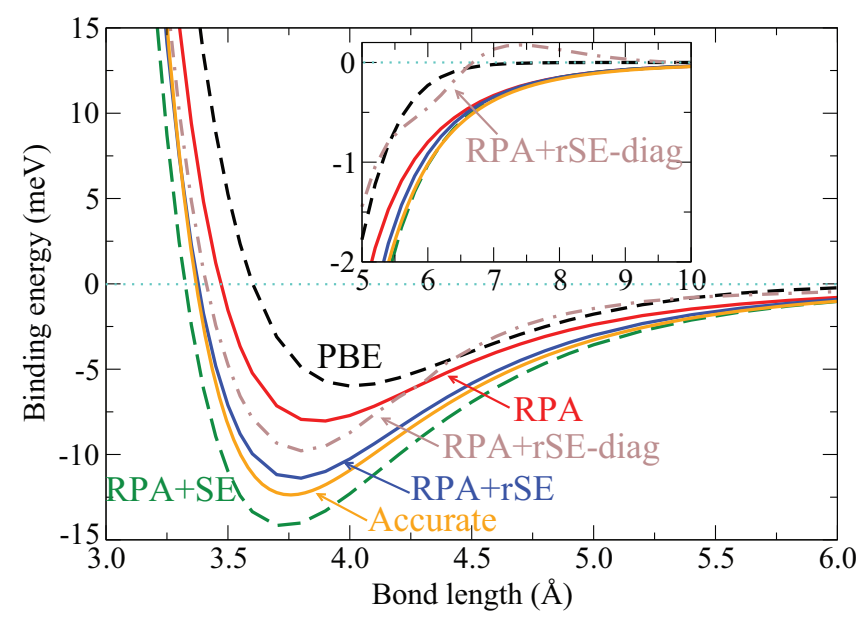

FIG. 4. (Color online) Binding-energy curves for $\mathrm{Ar}_{2}$ computed with PBE and RPA-based approaches (standard RPA, RPA + SE, $\mathrm{RPA}+\mathrm{rSE}$, and RPA + rSE-diag based on PBE), in comparison with the accurate Tang-Toennies (experimentally derived) curve. The results are obtained using the Gaussian "aug-cc-pV6Z" (Ref. 73) basis set. The basis set superposition error (BSSE) is corrected here and in all following calculations using the counterpoise correction scheme (Ref. 74). 


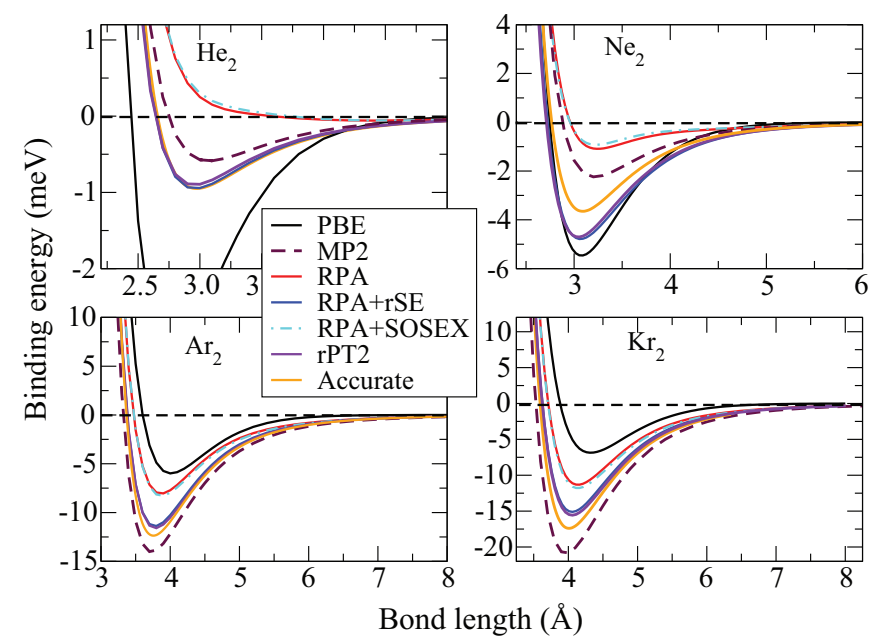

FIG. 5. (Color online) Binding-energy curves for rare-gas dimers computed with RPA-based approaches, in comparison with PBE, MP2, and the accurate Tang-Toennies reference curves. $\mathrm{He}_{2}, \mathrm{Ne}_{2}$, and $\mathrm{Ar}_{2}$ results are obtained using the aug-cc-pV6Z basis set, and $\mathrm{Kr}_{2}$ using the aug-cc-pV5Z basis set. All RPA-type calculations are based on the PBE reference.

eters. This model can accurately reproduce empirical data ${ }^{70}$ and agrees excellently with high-level quantum-chemical, e.g., $\operatorname{CCSD}(\mathrm{T})$ calculations. ${ }^{71,72}$ Coming back to the rSE discussion, the pathological behavior is thus caused by the diagonal approximation, and not inherent to the rSE scheme itself. In the remainder of our discussion on weakly interacting systems, we therefore only present results for the upgraded $\mathrm{RPA}+\mathrm{rSE}$ and rPT2 schemes.

The full set of binding energy curves for $\mathrm{He}_{2}, \mathrm{Ne}_{2}, \mathrm{Ar}_{2}$, and $\mathrm{Kr}_{2}$ obtained with PBE, MP2, RPA, rPT2, as well as the "intermediate" schemes RPA + rSE and RPA + SOSEX are then shown in Fig. 5. PBE does not contain long-range dispersion interactions by construction, and therefore decays too fast at large separations. Around the equilibrium region, PBE vastly overbinds $\mathrm{He}_{2}$ and $\mathrm{Ne}_{2}$, and underbinds $\mathrm{Ar}_{2}$ and $\mathrm{Kr}_{2}$. MP2 shows the opposite trend, although it performs better at a quantitative level. RPA systematically underbinds all dimers. This underbinding is most significant for $\mathrm{He}_{2}$ and $\mathrm{Ne}_{2}$. Adding the rSE correction leads to a substantial improvement for all dimers. With the largest available Dunning Gaussian basis sets ${ }^{73}$ (aug-cc-pV6Z for He, Ne, Ar and aug-cc-pV5Z for $\mathrm{Kr}$ ), RPA + rSE shows nearly perfect agreement with the reference curve for $\mathrm{He}_{2}$, overshoots a little bit for $\mathrm{Ne}_{2}$, and slightly underbinds $\mathrm{Ar}_{2}$ and $\mathrm{Kr}_{2}$. The SOSEX correction, on the other hand, has very little effect on the binding energies of these purely dispersion-bonded systems. As a result, rPT2 lies almost on top of RPA + rSE. The overall accuracy of $\mathrm{RPA}+\mathrm{rSE}$ and $\mathrm{rPT} 2$ for rare-gas dimers is very satisfactory, in particular since no adjustable parameters are used in these schemes.

\section{S22 and S66 test sets}

A widely used benchmark set for weak interactions are the S22 molecular complexes designed by Jurečka et al., ${ }^{36}$ for which accurate reference interaction energies obtained

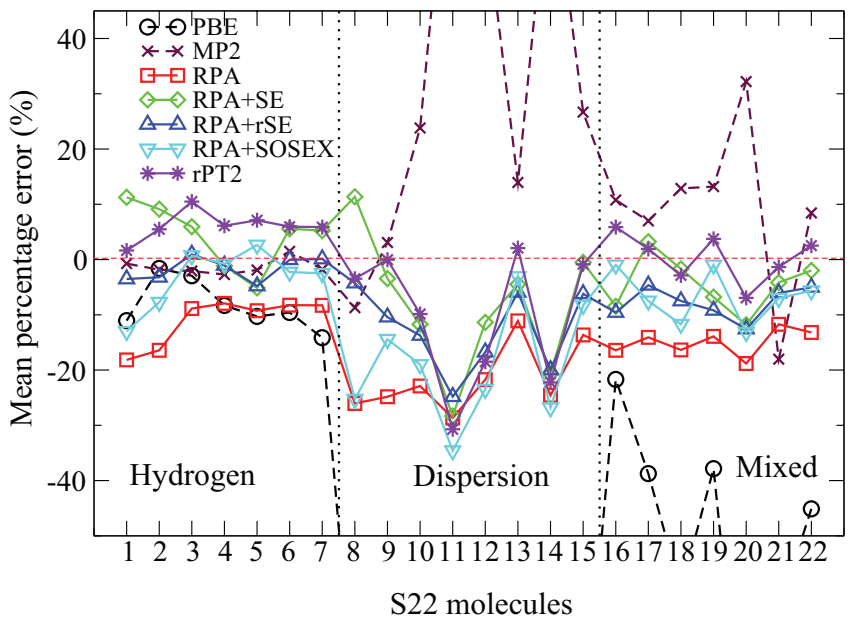

FIG. 6. (Color online) The percentage errors for the S22 test set for RPA-derived computational schemes (based on PBE reference orbitals), in comparison to PBE and MP2. The CCSD(T)/CBS results of Takatani et al. (Ref. 75) are used as reference. Lines are guides to the eye.

using the $\operatorname{CCSD}(\mathrm{T})$ method are available. ${ }^{75}$ This molecular test set includes the most common types of noncovalent interactions: hydrogen bonding, dispersion-dominated bonding, and mixed character. The performance of RPA and some of the RPA-related methods have been benchmarked for this test set. ${ }^{21,23,68,76}$ Similar to correlated quantum chemical methods, the quality of basis sets for RPA calculations is a significant issue. ${ }^{11,76,77}$ Using our numerical atomic orbital (NAO) tier-4 basis plus additional diffuse Gaussian functions from the aug-cc-pV5Z set [denoted as "tier 4+a5Z-d" (Ref. 44); see also Appendix D], we obtained a mean absolute error (MAE) of $0.90 \mathrm{kcal} / \mathrm{mol}$ in RPA@PBE for S22, fairly close to the $0.79 \mathrm{kcal} / \mathrm{mol}$ reported by Eshuis and Furche ${ }^{76}$ using Dunning's Gaussian basis sets extrapolated to the complete basis set (CBS) limit. In Appendix D, the convergence behavior of these two types of basis sets is shown for the methane dimer. In this work, we will continue to use the "tier $4+$ a5Z-d" basis set, bearing in mind that the absolute numbers could carry an uncertainty of $0.1 \mathrm{kcal} / \mathrm{mol}(4 \mathrm{meV})$, which will however not affect our discussion here.

In Fig. 6, the relative errors from RPA $+\mathrm{rSE}$, RPA + SOSEX, and rPT2 are presented for each individual molecule of the S22 set. Results from RPA and RPA + SE, as well as from PBE and MP2, are also included for comparison. PBE and MP2 are both performing well for hydrogen-bonded molecules where the electrostatic interactions dominate, but PBE underbinds the dispersion-dominated and those of mixedcharacter significantly, while the opposite is true for MP2. RPA-based methods are performing much better than PBE and MP2 for these two types of interactions. RPA + rSE falls between RPA and RPA + SE, although it lies closer to RPA + SE. For hydrogen-bonded molecules, RPA + rSE improves over RPA + SE, with the latter overbinding these molecules noticeably. Moreover, it is interesting to note that RPA + SOSEX improves over RPA appreciably for hydrogen and mixed bonding, but much less so for dispersion-bonded molecules. This is consistent with its performance for rare-gas 


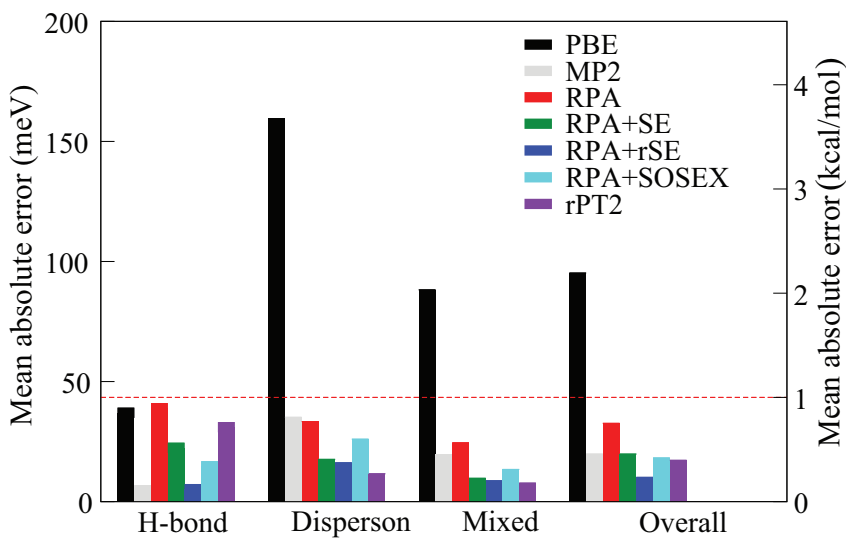

FIG. 7. (Color online) MAEs (in both $\mathrm{meV}$ and $\mathrm{kcal} / \mathrm{mol}$ ) for the S66 test set given by RPA, rPT2, and related schemes (based on PBE reference orbitals), in addition to PBE and MP2. The CCSD(T) results of Rezac et al. (Ref. 38) at the CBS limit are used here as reference.

dimers. Now, considering both rSE and SOSEX, together with RPA, rPT2 performs equally well or better for dispersiondominated and mixed bonding, but overshoots significantly for hydrogen bonding. So far, this is the only case we have found for which combining rSE and SOSEX worsens the description. Empirically, this is because both SOSEX and rSE improve the underbinding of RPA for hydrogen bonds. In particular, $\mathrm{RPA}+\mathrm{rSE}$ itself is already almost perfect, and adding further SOSEX correction overshoots. It is not entirely clear why this is so only from theoretical considerations. We hence document the problem here and hope to have more insights in future work. Finally, we note that for $\pi$-stacked systems such as the benzene dimer (No. 11 in Fig. 6), RPA gives a substantial error, but neither rSE nor SOSEX noticeably improves upon RPA. This warrants further attention in future studies.

Recently, the S22 test set has been extended to an even larger, more comprehensive, and balanced test set called S66. ${ }^{38}$ This overcomes several shortcomings of S22, e.g., the strong bias towards nucleic-acid-like structures. We also performed benchmark calculations with RPA, rPT2, and related schemes for this test set, and the results are presented in Fig. 7. The overall performance for S66 is very similar to that observed for S22. In brief, RPA + rSE performs better (or slightly better) than RPA + SE, which itself is a significant improvement over the standard RPA method. With the addition of the SOSEX term, the rPT2 approach performs even (slightly) better than $\mathrm{RPA}+\mathrm{rSE}$ for dispersion and mixed interactions. However, this is not the case for hydrogen bonds, where rPT2 clearly overshoots and the strength of hydrogen bonds becomes overestimated. Overall, for weak interactions, RPA $+\mathrm{rSE}$ outperforms other computational schemes benchmarked here, and yields a MAE of $10.1 \mathrm{meV}$ (or $0.23 \mathrm{kcal} / \mathrm{mol}$ ).

The molecular complexes of the S22 and S66 test sets are at their equilibrium geometries. Consequently, any conclusions drawn from these test sets (concerning the performance of RPA-based approaches) in principle only holds for these equilibrium geometries. However, this does not tell us anything about the performance of the RPA-based approaches for nonequilibrium geometries, which has important implications

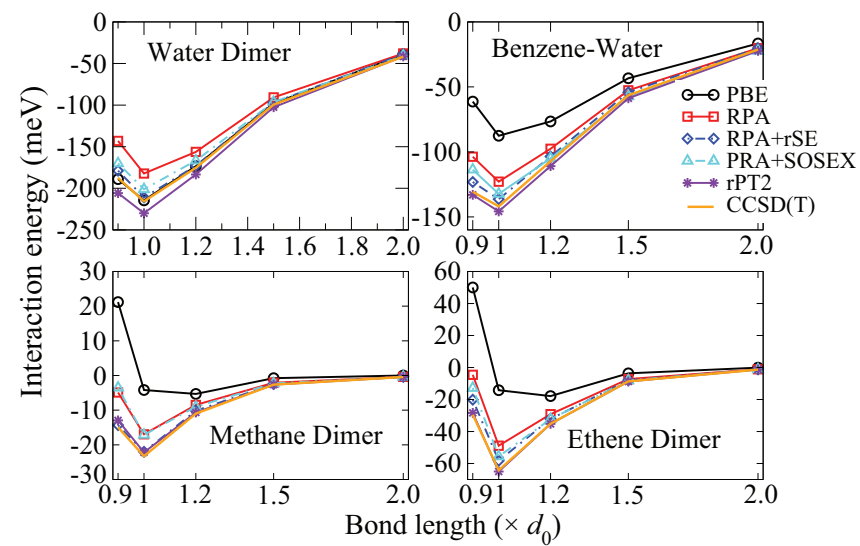

FIG. 8. (Color online) Interaction energies of four molecular dimers with distorted geometries. The data points correspond to shortening or elongating of the dimer along the main noncovalent interaction coordinate (at $0.9,1.0,1.2,1.5$, and 2.0 of the equilibrium distance). Geometries and reference $\operatorname{CCSD}(\mathrm{T})$ data are taken from the $\mathrm{S} 22 \times 5$ test set (Ref. 78 ).

for employing these approaches in molecular dynamics and/or applying them to big molecules whose building fragments are usually in a nonequilibrium situation. Therefore, here we check the performance of RPA and its variants for four molecular dimers from the S22 set as a function of the monomer separation. Reference $\operatorname{CCSD}(\mathrm{T})$ data are taken from the $\mathrm{S} 22 \times 5$ test set. $^{78}$ In Fig. 8 , we present the interaction energies of the four dimers at five different separations: the equilibrium distance, and four others stretched along the main interaction coordinate while keeping the monomer geometry unchanged. Figure 8 illustrates that there is no significant difference in the performance of the RPA-based methods at the different distances. Thus, the conclusions drawn from the S22 and S66 benchmark studies carry over to large molecules and/or molecules with distorted geometries.

\section{B. Atomization energies}

The atomization energy of molecules is a key quantity in thermochemistry. RPA has been tested for this quantity in early works, ${ }^{11,24}$ where a pronounced underbinding behavior was observed. In a recent work, Paier et al ${ }^{35}$ reported a detailed study of the atomization energies of the G2-I set ${ }^{39}$ using RPA and its variants, including the rPT2-diag scheme as discussed before. To test the influence of the off-diagonal elements of rSE in the rPT2 scheme, we present in Fig. 9 the MAEs for RPA, rPT2-diag, rPT2, and related methods. Some of these results were already included in our recent review paper on RPA. ${ }^{10}$ In brief, both the (r)SE and SOSEX corrections reduce the underbinding trend of RPA significantly. Combining the rSE and SOSEX corrections, the two terms work collaboratively and the resultant $\mathrm{rPT} 2$ method reduces the MAE further by a factor of 2 . In contrast to the nonbonded interactions discussed in the previous section, the difference between $\mathrm{rPT} 2$ and rPT2-diag is small $(0.18 \mathrm{kcal} / \mathrm{mol}$ or $8 \mathrm{meV}$ difference in MAE), validating our previous conclusions regarding the atomization energies in Ref. 35 that were based on the rPT2-diag scheme. 


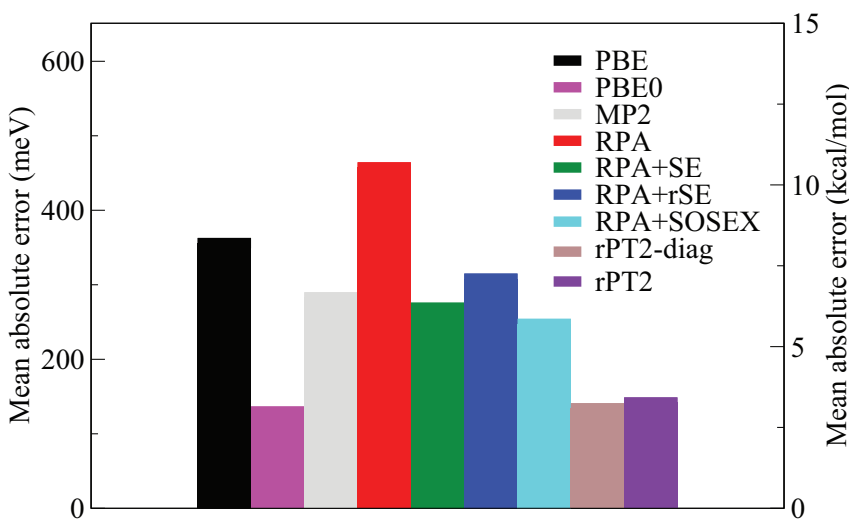

FIG. 9. (Color online) The MAEs (in both $\mathrm{meV}$ and $\mathrm{kcal} / \mathrm{mol}$ ) of the G2-I atomization energies (Ref. 39) obtained with PBE, MP2, RPA, rPT2, and related methods. The Gaussian cc-pV6Z basis set (Ref. 73) was used in all calculations. Reference data are from Ref. 79.

In this context we would like to warn that, despite the success of RPA + SOSEX and, in particular, rPT2 for describing the atomization energies on average, for certain molecules (in particular $\mathrm{O}_{2}$ and $\mathrm{N}_{2}$ ), the SOSEX term worsens the results, leading to more pronounced underestimation of binding energies. A detailed investigation of this issue is beyond the scope of this paper, and will be carried out in future work.

One of the motivations for developing the rSE scheme over the intial SE term was to describe systems with metallic character, for which a simple second-order SE cannot be applied. It is thus interesting to see how RPA + rSE and rPT2 perform for metallic clusters (and eventually bulk metals). While a systematic investigation of this issue is still ongoing, we can present here some preliminary results for small copper clusters for illustrative purposes. In Fig. 10, we plot the effective cohesive energy as a function of the cluster size. The effective cohesive energy ( $\left.E_{\mathrm{coh}}\right)$ is defined as $E_{\mathrm{AE}} / C_{\text {eff }}$ where $E_{\mathrm{AE}}$ is the atomization energy of the cluster, and $\left.C_{\text {eff }}=\sum_{i} \sqrt{(} C_{i} / 12\right)$ is the effective coordination number of the cluster, with $C_{i}$ being the number of nearest neighbors of atom $i{ }^{80}$ The copper clusters chosen in this study are somewhat artificial in the sense that they were cut from bulk copper at the PBE lattice constant of $3.633 \AA$. $E_{\text {coh }}$ approaches the bulk cohesive energy of copper in the limit of sufficiently large cluster sizes. Although the clusters considered in this work are still too small to extract this bulk limit reliably, they suffice to establish a first idea of the functional quality. For comparison, the experimental reference cohesive energy for bulk copper is 3.49 eV. ${ }^{81}$ From Fig. 10 we can see that RPA itself yields cohesive energies that are too small and close to the PBE0 values. This is consistent with the general underbinding trend of RPA. Adding the SOSEX correction has very little effect, increasing the cohesive energy only by about $50 \mathrm{meV}$. In contrast, the rSE term significantly increases the cohesive energy by about $1 \mathrm{eV}$. Now, the resultant RPA $+\mathrm{rSE}$ and rPT2 cohesive energies are approximately halfway between LDA and PBE. This is in general a good sign since for most solids the experimental cohesive energy lies between the LDA and PBE ones. For $\mathrm{Cu}$, on the other hand, this indicates an overbinding in rPT2 since in this case the PBE value is
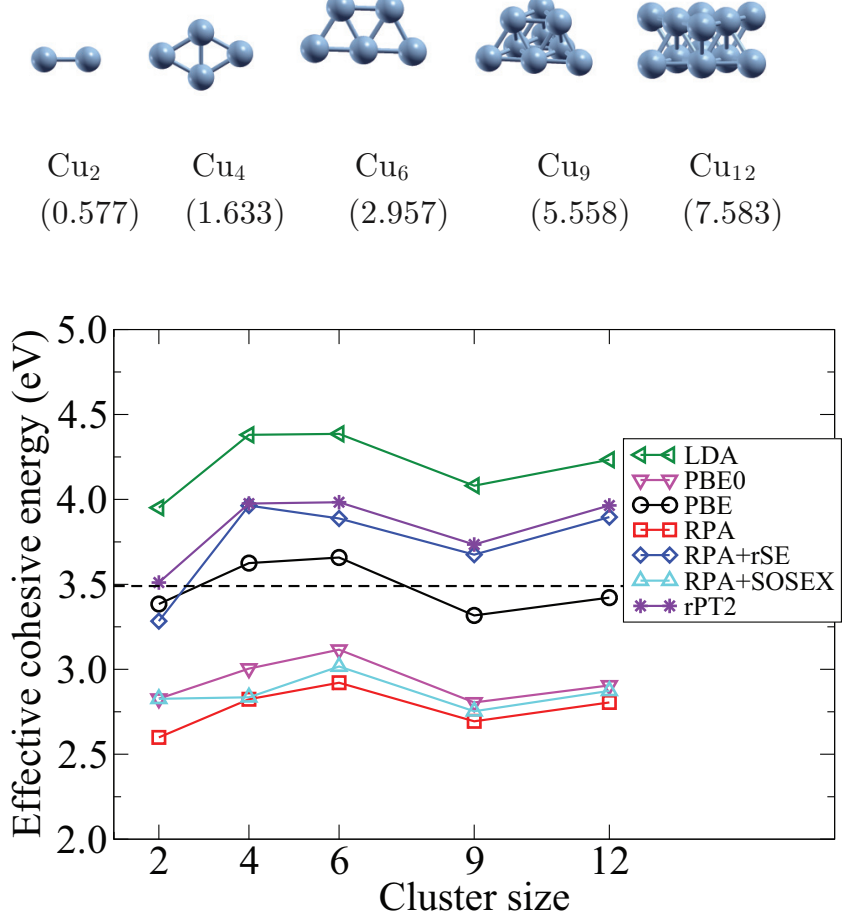

FIG. 10. (Color online) Upper panel: structure of the $\mathrm{Cu}$ clusters cut from the $\mathrm{Cu}$ bulk. The number in parentheses corresponds to the effective coordination number $C_{\text {eff }}$ of that cluster. Lower panel: effective cohesive energy for the $\mathrm{Cu}$ clusters obtained using different approaches. The dashed line marks the experimental cohesive energy for copper bulk (Ref. 81). The FHI-AIMS tier-4 basis set was used. All RPA-based calculations were done with a PBE reference.

very close to the experimental one. To draw more general conclusions for metallic systems, more comprehensive and systematic benchmark studies are needed.

\section{Equilibrium geometry of diatomic molecules}

Another important criterion for assessing the quality of electronic-structure methods are the equilibrium geometries they predict. In Table I we present the calculated equilibrium bond lengths of 10 diatomic molecules (denoted as EBL10 set in the following). The EBL10 set contains prototypical molecules of covalent, ionic, and vdW bonding type, and hence can be regarded as a test set with some representative character. From Table I we can conclude that the conventional functionals PBE and PBE0 perform well for covalent and ionic bonds, but significantly overestimate the bond lengths of rare-gas dimers. This is not surprising since they do not properly account for dispersion forces. For alkali-metal dimers, these two functionals describe $\mathrm{Na}_{2}$ very well but not $\mathrm{Li}_{2}$. As for MP2, its performance is similar to PBE and PBE0, except for the rare-gas dimers for which MP2 gives a significant improvement, although the bond-length underestimation of $\mathrm{NeAr}$ and the overestimation of $\mathrm{Ar}_{2}$ are still sizable.

The behavior of RPA closely resembles PBE for covalent and ionic bonds, but differs for the metal dimers. Here, RPA does surprisingly well for $\mathrm{Li}_{2}$ (in contrast to all other 
TABLE I. Equilibrium bond lengths (in $\AA$ ) for 10 diatomic molecules. Errors with respect to experimental numbers are given in parentheses. The reference values for $\mathrm{Ar}_{2}$ and $\mathrm{NeAr}$ are taken from Ref. 70, and the rest from Ref. 82 .

\begin{tabular}{|c|c|c|c|c|c|c|c|c|}
\hline & PBE & PBE0 & MP2 & RPA & $\mathrm{RPA}+\mathrm{rSE}$ & RPA + SOSEX & $\mathrm{rPT} 2$ & Expt \\
\hline $\mathrm{CO}$ & $1.135(0.007)$ & $1.122(-0.006)$ & $1.131(0.003)$ & $1.135(0.007)$ & $1.143(0.015)$ & $1.117(-0.011)$ & $1.123(-0.005)$ & 1.128 \\
\hline $\mathrm{O}_{2}$ & $1.218(0.010)$ & $1.191(-0.017)$ & $1.213(0.005)$ & $1.222(0.014)$ & $1.232(0.024)$ & $1.175(-0.033)$ & $1.183(-0.025)$ & 1.208 \\
\hline $\mathrm{N}_{2}$ & $1.102(0.004)$ & $1.089(-0.009)$ & $1.108(0.010)$ & $1.103(0.005)$ & $1.107(0.009)$ & $1.083(-0.015)$ & $1.087(-0.011)$ & 1.098 \\
\hline $\mathrm{HF}$ & $0.930(0.013)$ & $0.920(0.003)$ & $0.918(0.001)$ & $0.922(0.005)$ & $0.927(0.010)$ & $0.909(-0.008)$ & $0.912(-0.005)$ & 0.917 \\
\hline $\mathrm{LiH}$ & $1.605(0.010)$ & $1.597(0.002)$ & $1.592(-0.003)$ & $1.594(-0.001)$ & $1.598(0.003)$ & $1.592(-0.003)$ & $1.596(0.001)$ & 1.595 \\
\hline $\mathrm{NaCl}$ & $2.379(0.018)$ & $2.368(0.007)$ & $2.373(0.012)$ & $2.379(0.018)$ & $2.384(0.023)$ & $2.373(0.012)$ & $2.377(0.016)$ & 2.361 \\
\hline $\mathrm{Li}_{2}$ & $2.729(0.056)$ & $2.726(0.053)$ & $2.724(0.051)$ & $2.680(0.007)$ & $2.695(0.022)$ & $2.716(0.043)$ & $2.727(0.054)$ & 2.673 \\
\hline $\mathrm{Na}_{2}$ & $3.087(0.008)$ & $3.092(0.013)$ & $3.095(0.016)$ & $3.105(0.026)$ & $3.127(0.048)$ & $3.098(0.019)$ & $3.118(0.039)$ & 3.079 \\
\hline $\mathrm{Ar}_{2}$ & $4.009(0.252)$ & $4.070(0.313)$ & $3.729(-0.028)$ & $3.866(0.109)$ & $3.771(0.014)$ & $3.871(0.114)$ & $3.776(0.019)$ & 3.757 \\
\hline $\mathrm{NeAr}$ & $3.538(0.062)$ & $3.608(0.132)$ & $3.542(0.066)$ & $3.606(0.130)$ & $3.461(-0.015)$ & $3.602(0.126)$ & $3.457(-0.019)$ & 3.476 \\
\hline $\mathrm{ME}$ & 0.044 & 0.049 & 0.013 & 0.032 & 0.015 & 0.024 & 0.006 & \\
\hline MAE & 0.044 & 0.056 & 0.020 & 0.032 & 0.018 & 0.038 & 0.019 & \\
\hline
\end{tabular}

methods), but not so well for $\mathrm{Na}_{2}$. For rare-gas dimers, the general underbinding trend shifts the binding-energy curves towards larger distances, resulting in significantly overestimated bond lengths. Adding the rSE correction to RPA shifts the equilibrium distances to larger values for all strong bonds, and the resultant bond lengths are slightly overestimated. For vdW bonds, on the other hand, RPA + rSE performs exceptionally well, bringing the equilibrium bond lengths into close agreement with the reference values. RPA + SOSEX, on the other hand, shows roughly the opposite trend for covalent and ionic bonds, but over-stretches the bond lengths of metal and vdW dimers. Finally, putting SOSEX and rSE corrections together, the rPT2 method shows an overall satisfactory description for covalent, ionic, and vdW bonds. Only for metal dimers the errors are noticeably larger. In terms of MAEs, MP2, RPA + rSE, and rPT2 are on a par with each other. For covalent and ionic bonds, MP2 is noticeably better. This is probably because for these molecules (with a wide gap) the HF reference represents a better starting point than PBE for perturbation theory. However, RPA + rSE and rPT2 are clearly better for vdW bonds. Thus, at this point no single approach

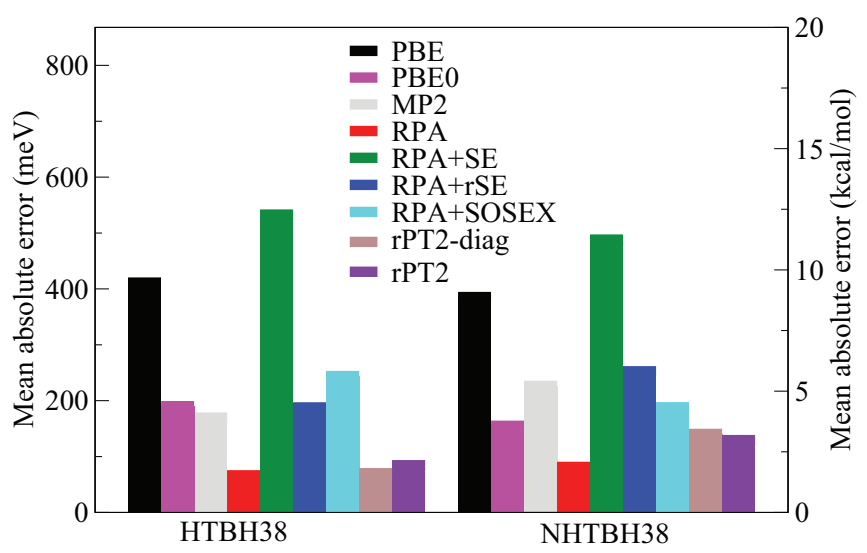

FIG. 11. (Color online) The MAEs (in both $\mathrm{meV}$ and $\mathrm{kcal} / \mathrm{mol}$ ) of the HTBH38 and NHTBH38 test sets for barrier heights, obtained with PBE, MP2, RPA, rPT2, and related methods (based on PBE). Reference data are from Refs. 40 and 41. Gaussian cc-pV6Z basis sets were used in the calculations. stands out as the best one for all bonding situations, although rPT2 gives the smallest ME and a good MAE.

\section{Barrier heights}

To complete our discussion, we address here chemical reaction barrier heights. For this purpose, we chose the HTBH38 and NHTBH38 test set of Truhlar and co-workers. ${ }^{40,41}$ RPAbased methods were benchmarked in previous studies $9,10,35$ and we here revisit this set with the upgraded version of rPT2. The MAEs for our different schemes are shown in Fig. 11. Standard RPA performs remarkably well for reaction barrier heights compared to all alternatives. This has been rationalized by Henderson and Scuseria ${ }^{47}$ to be due to the inherent self-correlation error in RPA that mimics "static correlation" [i.e., the (near) degeneracy of two (or more) determinants], leading to an excellent description of the transition states due to partial error cancellation. Unfortunately, any attempt to correct RPA deteriorates its performance in this case. In particular, the RPA + SE method provides a bad description of the transition states, resulting in errors that are even larger than in PBE. The RPA + SE error reduces when the SE term is renormalized in RPA + rSE. Now, in contrast to the G2-I case, the errors in RPA + rSE and RPA + SOSEX point in opposite directions and tend to cancel each other. Again, by combining the two schemes, rPT2 shows a better performance than separate RPA + rSE and RPA + SOSEX schemes, and gives a much more satisfactory description of the barrier heights. Similar to the G2-I test set, the difference between rPT2 and rPT2-diag is small $(0.33 \mathrm{kcal} / \mathrm{mol}$ for HTBH38 and $0.25 \mathrm{kcal} / \mathrm{mol}$ for NHTBH38 in MAE) compared to the variation among other schemes.

\section{CONCLUSIONS}

In summary, the rPT2 method comprises a systematic renormalization of all three terms that appear in second-order perturbation theory, through an infinite summation of three distinct series of diagrams. The resulting three terms in rPT2 are RPA, SOSEX, and rSE. In this work, we formulated an alternative way to express the SOSEX correlation energy, discussed in detail how to sum up the "off-diagonal" elements 
TABLE II. Error statistics of rPT2 and related approaches for the S22, G2, EBL10, and NHTBH38/HTBH38 test sets. Listed are the MAPE for S22, and the MAE for the other sets.

\begin{tabular}{lrcccc}
\hline \hline Method & $\begin{array}{r}\text { S22 } \\
(\%)\end{array}$ & $\begin{array}{c}\text { G2 } \\
(\mathrm{kcal} / \mathrm{mol})\end{array}$ & $\begin{array}{c}\text { EBL10 } \\
(\AA)\end{array}$ & $\begin{array}{c}\mathrm{HTBH} 88 \\
(\mathrm{kcal} / \mathrm{mol})\end{array}$ & $\begin{array}{c}\text { NHTBH38 } \\
(\mathrm{kcal} / \mathrm{mol})\end{array}$ \\
\hline PBE & 57.8 & 8.2 & 0.044 & 9.7 & 8.9 \\
PBE0 & 55.2 & 3.0 & 0.056 & 4.4 & 3.6 \\
MP2 & 18.7 & 6.5 & 0.020 & 3.9 & 5.2 \\
RPA & 16.1 & 10.5 & 0.032 & 1.5 & 1.9 \\
RPA + rSE & 7.7 & 7.1 & 0.018 & 4.3 & 5.8 \\
RPA + SOSEX & 10.5 & 5.7 & 0.038 & 5.6 & 4.3 \\
rPT2 & 7.1 & 3.1 & 0.019 & 1.9 & 3.0 \\
\hline \hline
\end{tabular}

in rSE, which were neglected in previous works, and illustrated the concept of rPT2 from a diagrammatic point of view. We benchmarked the performance of $\mathrm{PPT} 2$ and related approaches (RPA + rSE, RPA + SOSEX), focusing on weakly interacting molecules. We found that rPT2 works well for dispersion and mixed-type interactions, but for hydrogen bonds it overcorrects the underbinding behavior of RPA. We also examined the influence of the previously neglected "off-diagonal" elements in the rSE correction and found that, for weak interactions, it is crucial to include them, whereas for atomization energy and reaction barrier heights, the off-diagonal elements only have a minor effect. We also found that the SOSEX correction improves the description of electrostatic interactions substantially, but has very little effect on dispersion interactions. rSE, on the other hand, leads to a better description of both electrostatic and dispersion interactions.

Overall, rPT2 provides a conceptually appealing, and diagrammatically systematic way for going beyond RPA. In Table II, we summarized the error statistics for the representative test sets considered in this work. It can be seen that, although rPT2 does not always deliver the best accuracy in every single case compared to other approaches, it provides "the most balanced" description across the various different electronic and chemical environments. We thus consider the rPT2 scheme as a natural step for extending and improving the RPA method. We trust that the successes and shortcomings of rPT2 documented in this work provide a useful basis for developing more accurate, robust, and generally applicable electronic-structure methods in the coming years.

\section{ACKNOWLEDGMENTS}

We thank J. Paier for providing his SOSEX numbers generated using GAUSSIAN, and J. E. Moussa for a critical reading of the manuscript and for pointing out the distinction between SOSEX and AC-SOSEX. The work at Rice University was supported by the US Department of Energy, Office of Basic Energy Sciences (Grant No. DEFG02-09ER16053) and the Welch Foundation (Grant No. C-0036).

\section{APPENDIX A: IMPLEMENTATION OF AC-SOSEX IN FHI-AIMS}

The RPA implementation in the FHI-AIMS code $^{43}$ has been described in detail in Ref. 44. Here, we will give a brief account of the SOSEX implementation in our code. The energy expression that we like to evaluate is

$$
\begin{aligned}
E_{c}^{\mathrm{AC}-S O S E X}= & -\frac{1}{2 \pi} \int_{0}^{\infty} d \omega \sum_{i a, j b}\langle i j \mid b a\rangle\langle i j|\bar{W}(i \omega)| a b\rangle \\
& \times \mathcal{F}_{i a}(i \omega) \mathcal{F}_{j b}(i \omega),
\end{aligned}
$$

where $\langle i j \mid b a\rangle$ are the two-electron Coulomb integrals defined in Eq. (5), and $\langle i j|\bar{W}(i \omega)| a b\rangle$ are the corresponding (coupling-constant-averaged) screened Coulomb integrals. The frequency-dependent factor $\mathcal{F}_{i a}(i \omega)$ is defined in Eq. (18).

In analogy to the RPA case, the basic technique to evaluate the two-electron integrals in our code is the resolution of identity. We chose the Coulomb metric, denoted "RI-V" in the following. Here, we would like to emphasize that "RI-V" is a highly accurate method, and the error incurred thereby is vanishingly small for practical purposes (see Ref. 44 for detailed benchmarks). In RI-V, the bare two-electron integrals are computed as

$$
\langle i j \mid a b\rangle=\sum_{\mu \nu}(i a \mid \mu) V_{\mu \nu}^{-1}(\nu \mid i b),
$$

where

$$
(i a \mid \mu)=\iint d \mathbf{r} d \mathbf{r}^{\prime} \frac{\psi_{i}(\mathbf{r}) \psi_{a}(\mathbf{r}) P_{\mu}\left(\mathbf{r}^{\prime}\right)}{\left|\mathbf{r}-\mathbf{r}^{\prime}\right|}
$$

and

$$
V_{\mu \nu}=\iint d \mathbf{r} d \mathbf{r}^{\prime} \frac{P_{\mu}(\mathbf{r}) P_{\nu}\left(\mathbf{r}^{\prime}\right)}{\left|\mathbf{r}-\mathbf{r}^{\prime}\right|} .
$$

Here, $\psi_{p}$ are canonical single-particle spin orbitals, and $P_{\mu}(\mathbf{r})$ are a set of suitably constructed auxiliary basis functions. ${ }^{44}$ For notational simplicity, all orbitals are assumed to be real.

In practice, we decompose the $V^{-1}$ matrix in Eq. (A2) into the product of its square roots, and combine each three-index integral with a square root. This gives

$$
\langle i j \mid a b\rangle=\sum_{\mu} O_{i a}^{\mu} O_{j b}^{\mu}
$$

with

$$
O_{i a}^{\mu}=\sum_{\nu}(i a \mid v) V_{\nu \mu}^{-1 / 2} .
$$

As discussed in the context of the $G W$ implementation in FHI-AIMS ${ }^{44}$ the "RI-V" technique can be used to treat the screened two-electron Coulomb integrals as well. In this case, we have

$$
\langle i j|\bar{W}(i \omega)| a b\rangle=\sum_{\mu, \nu} O_{i a}^{\mu} \overline{\mathcal{E}}_{\mu \nu}^{-1}(i \omega) O_{j b}^{\nu},
$$

where $\overline{\mathcal{E}}$ is the coupling-constant-averaged dielectric functions, formally linked to the screened Coulomb matrix by

$$
\overline{\mathcal{E}}^{-1}(i \omega)=V^{-1 / 2} \bar{W}(i \omega) V^{-1 / 2} .
$$

In Eq. (A8), $\bar{W}(i \omega)$ is the screened Coulomb interaction matrix represented in terms of the auxiliary basis set,

$$
\bar{W}_{\mu \nu}(i \omega)=\iint d \mathbf{r} d \mathbf{r}^{\prime} P_{\mu}(\mathbf{r}) \bar{W}_{\mu \nu}\left(\mathbf{r}, \mathbf{r}^{\prime}, i \omega\right) P_{\nu}\left(\mathbf{r}^{\prime}\right)
$$

For convenience, we introduce a quantity $\Pi(i \omega)=$ $v^{1 / 2} \chi_{0}(i \omega) v^{1 / 2}$, where $\chi_{0}(i \omega)$ is the independent density 
response function defined in Eq. (2). Using Eqs. (2), (A3), and (A6), one can easily obtain the matrix representation of $\Pi(i \omega)$ in the auxiliary basis

$$
\Pi_{\mu \nu}(i \omega)=\sum_{i a} \frac{2\left(\epsilon_{i}-\epsilon_{a}\right)}{\omega^{2}+\left(\epsilon_{i}-\epsilon_{a}\right)^{2}} O_{i a}^{\mu} O_{i a}^{v},
$$

where $\epsilon_{i}$ and $\epsilon_{a}$ are occupied and unoccupied single-particle orbital energies, respectively. Using Eq. (14), the matrix form of $\overline{\mathcal{E}}^{-1}$ becomes

$$
\overline{\mathcal{E}}^{-1}(i \omega)=\int_{0}^{1} d \lambda[1-\lambda \Pi(i \omega)]^{-1} \lambda .
$$

The $\lambda$ integration in Eq. (A11) can be accurately computed using a Gauss-Legendre quadrature with 5-6 grid points.

Combining Eqs (A1), (A5), and (A7), the final expression for the RI-SOSEX energy is

$$
\begin{aligned}
E_{\mathrm{c}}^{\mathrm{SOSEX}}= & -\frac{1}{2 \pi} \int_{0}^{\infty} d \omega \sum_{i j, a b}\left[\left(\sum_{\mu} O_{i a}^{\mu} O_{j b}^{\mu}\right)\right. \\
& \left.\times\left(\sum_{\nu \gamma} O_{i a}^{v} \overline{\mathcal{E}}_{\nu \gamma}^{-1}(i \omega) O_{j b}^{\gamma}\right)\right] \mathcal{F}_{i a}(i \omega) \mathcal{F}_{j b}(i \omega) .
\end{aligned}
$$

The computational effort for evaluating Eq. (A12) formally scales as $O\left(N^{5}\right)$, where $N$ is the system size.

\section{APPENDIX B: COMPARISON OF SOSEX AND AC-SOSEX}

In Table III, we present the RPA and SOSEX correlation energies ( $E_{\mathrm{c}}^{\mathrm{RPA}}$ and $\left.E_{\mathrm{c}}^{\mathrm{SOSEX}}\right)$, as well as the RPA and $\mathrm{RPA}+\mathrm{SOSEX}$ atomization energies for five molecules. The vanishingly small differences in the RPA energies are due to the different implementations in FHI-AIMS and the development version of the GAUSSIAN (Ref. 83) code (e.g., FHI-AIMS employs the RI approximation and treats the Gaussian orbitals numerically). The difference in the SOSEX and AC-SOSEX correlation energies reflects the intrinsic differences of the two SOSEX formulations. Nevertheless, the differences are very small and have little practical importance, in particular for atomization energies.

\section{APPENDIX C: DERIVATION OF THE RENORMALIZED SINGLE-EXCITATION (rSE) CONTRIBUTION}

We start with the expression for the second-order singleexcitation (SE) contribution

$$
E_{\mathrm{c}}^{\mathrm{SE}}=\sum_{i, a} \frac{\left\langle\Phi_{0}\left|\hat{H}^{\prime}\right| \Phi_{i}^{a}\right\rangle\left\langle\Phi_{i}^{a}\left|\hat{H}^{\prime}\right| \Psi_{0}\right\rangle}{E_{0}^{(0)}-E_{i a}^{(0)}},
$$

where $\hat{H}^{\prime}=\hat{H}-\hat{H}_{0}$ with $\hat{H}_{0}$ being a sum of single-particle (Kohn-Sham) Hamiltonian operators: $\hat{H}_{0}=\sum_{i} \hat{h}^{0}(i) . E_{0}^{(0)}$ and $E_{i a}^{(0)}$ are eigenenergies of the ground state $\left|\Psi_{0}\right\rangle$ and singly excited states $\left|\Psi_{i a}\right\rangle$ of $\hat{H}_{0}$, respectively.

The form of this equation actually already implies that the singly excited states $\left|\Phi_{i}^{a}\right\rangle$ are Slater determinants composed of canonical orbitals, namely, $\left|\Psi_{i}^{a}\right\rangle=\operatorname{Det}\left\{\psi_{q}\right\}$ where $\hat{h}^{0}\left|\psi_{q}\right\rangle=$ $\epsilon_{q}\left|\psi_{q}\right\rangle$, and $\hat{H}_{0}\left|\Phi_{i}^{a}\right\rangle=E_{i a}^{(0)}\left|\Phi_{i}^{a}\right\rangle$ with $E_{i a}^{(0)}=E_{0}^{(0)}+\epsilon_{a}-\epsilon_{i}$. Equation $(\mathrm{C} 1)$ can be reduced to an expression in terms of (canonical) single-particle orbitals ${ }^{84}$

$$
E_{\mathrm{c}}^{\mathrm{SE}}=\sum_{i, a} \frac{\left|\left\langle\psi_{i}|\hat{f}| \psi_{a}\right\rangle\right|^{2}}{\epsilon_{i}-\epsilon_{a}}=\sum_{i, a} \frac{\left|f_{i a}\right|^{2}}{\epsilon_{i}-\epsilon_{a}},
$$

TABLE III. RPA and SOSEX (total) correlation energies, as well as RPA and RPA + SOSEX atomization energies for five molecules. The "AC-SOSEX" numbers are computed using FHI-AIMS based on Eq. (19), whereas the original ring-CCD based SOSEX numbers are computed

\begin{tabular}{|c|c|c|c|c|c|c|}
\hline & \multirow{2}{*}{\multicolumn{3}{|c|}{ RPA }} & \multicolumn{3}{|c|}{ AC-SOSEX/SOSEX } \\
\hline & & & & \multirow{2}{*}{$\begin{array}{c}\text { FHI-AIMS } \\
(\mathrm{AC}-\mathrm{SOSEX})\end{array}$} & \multirow{2}{*}{$\begin{array}{l}\text { GAUSSIAN } \\
\text { (SOSEX) }\end{array}$} & \multirow[b]{2}{*}{ Difference } \\
\hline & FHI-AIMS & GAUSSIAN & Difference & & & \\
\hline $\mathrm{N}_{2}$ & -16.5001 & -16.5007 & 0.0006 & 6.0972 & 6.0669 & 0.0303 \\
\hline $\mathrm{O}_{2}$ & -19.8738 & -19.8742 & 0.0004 & 7.7113 & 7.6484 & 0.0629 \\
\hline $\mathrm{CH}_{4}$ & -10.3875 & -10.3874 & -0.0001 & 4.2243 & 4.2159 & 0.0084 \\
\hline \multirow[t]{2}{*}{$\mathrm{C}_{2} \mathrm{H}_{2}$} & -14.6788 & -14.6789 & 0.0002 & 5.6422 & 5.6195 & 0.0227 \\
\hline & & & Atomi & & & \\
\hline
\end{tabular}
using a development version of the GAUSSIAN (Ref. 83) suite of programs. All calculations were done with Gaussian cc-pVQZ basis set and

\begin{tabular}{|c|c|c|c|c|c|c|}
\hline & \multirow{2}{*}{\multicolumn{3}{|c|}{ RPA }} & \multicolumn{3}{|c|}{ RPA + AC-SOSEX/RPA + SOSEX } \\
\hline & & & & \multirow{2}{*}{$\begin{array}{c}\text { FHI-AIMS } \\
\text { (AC-SOSEX) }\end{array}$} & \multirow{2}{*}{$\begin{array}{r}\text { GAUSSIAN } \\
\text { (SOSEX) }\end{array}$} & \multirow[b]{2}{*}{ Difference } \\
\hline & FHI-AIMS & GAUSSIAN & Difference & & & \\
\hline$\overline{\mathrm{CO}}$ & 10.3712 & 10.3721 & -0.0009 & 10.7060 & 10.7051 & 0.0009 \\
\hline $\mathrm{N}_{2}$ & 9.4354 & 9.4358 & -0.0004 & 9.0737 & 9.0676 & 0.0061 \\
\hline $\mathrm{O}_{2}$ & 4.6843 & 4.6847 & -0.0004 & 4.2546 & 4.2806 & -0.0260 \\
\hline $\mathrm{CH}_{4}$ & 17.3526 & 17.3517 & 0.0009 & 18.0108 & 18.0091 & 0.0017 \\
\hline $\mathrm{C}_{2} \mathrm{H}_{2}$ & 16.1938 & 16.1947 & -0.0009 & 16.9831 & 16.9866 & -0.0035 \\
\hline
\end{tabular}
frozen-core $(1 s)$ approximation. The reference orbitals are obtained using the GGA-PBE functional. Note that in the upper part of the table only the RPA or (AC-)SOSEX correlation contribution is included, whereas in the lower part the numbers are obtained from the total energy (including also the Hartree-Fock part) differences.

Correlation energy $(\mathrm{eV})$ 
where $\hat{f}$ is the single-particle Hartree-Fock operator. Here we have adhered the common convention that $i, j, k, \ldots$ denote occupied single-particle orbitals, $a, b, c, \ldots$ unoccupied orbitals, and $p, q, r, \ldots$ for general cases.
To set the stage for later discussions, we can also more generally express the SE energy in Eq. (C1) in terms of noncanonical orbitals $\left\{\chi_{q}\right\}$, where $\hat{h}^{0}\left|\chi_{p}\right\rangle=\sum_{q} h_{p q}^{0}\left|\chi_{q}\right\rangle$ and $h_{p q}^{0}=\left\langle\chi_{p}\left|\hat{h}^{0}\right| \chi_{q}\right\rangle$. In this case, $E_{\mathrm{c}}^{\mathrm{SE}}$ is given by

$$
E_{\mathrm{c}}^{\mathrm{SE}}=\sum_{i j, a b}\left\langle\Phi_{0}\left|\hat{H}^{\prime}\right| \Phi_{i}^{a}\right\rangle\left\langle\Phi_{i}^{a}\left|\left(E_{0}^{(0)}-\hat{H}_{0}\right)^{-1}\right| \Phi_{j}^{b}\right\rangle\left\langle\Phi_{j}^{b}\left|\hat{H}^{\prime}\right| \Phi_{0}\right\rangle=\sum_{i j, a b}\left\langle\chi_{i}|\hat{f}| \chi_{a}\right\rangle\left[\left(E_{0}^{(0)} I-H_{0}\right)^{-1}\right]_{i a, j b}\left\langle\chi_{b}|\hat{f}| \chi_{j}\right\rangle,
$$

where $I$ is the identity matrix $I_{i a, j b}=\delta_{i j} \delta_{a b}$, and

$$
\left[E_{0}^{(0)} I-H_{0}\right]_{i a, j b}=\left\langle\Phi_{i}^{a}\left|E_{0}^{(0)}-\hat{H}_{0}\right| \Phi_{j}^{b}\right\rangle=h_{i j}^{0} \delta_{a b}-h_{a b}^{0} \delta_{i j} .
$$

Now, the question arises how to sum up all the higher-order SE diagrams shown in Fig. 2? For canonical orbitals, the corresponding algebraic expression can be easily obtained by applying the rules of evaluating Goldstone diagrams ${ }^{56}$ :

$$
\begin{aligned}
E_{\mathrm{c}}^{\mathrm{rSE}}= & \sum_{i a} \frac{f_{a i} f_{i a}}{\epsilon_{i}-\epsilon_{a}}-\sum_{i j, a} \frac{f_{a i} \Delta v_{i j} f_{j a}}{\left(\epsilon_{i}-\epsilon_{a}\right)\left(\epsilon_{j}-\epsilon_{a}\right)}+\sum_{i, a b} \frac{f_{a i} f_{i b} \Delta v_{b a}}{\left(\epsilon_{i}-\epsilon_{a}\right)\left(\epsilon_{i}-\epsilon_{b}\right)}+\sum_{i j k, a} \frac{f_{a i} \Delta v_{i k} \Delta v_{k j} f_{j a}}{\left(\epsilon_{i}-\epsilon_{a}\right)\left(\epsilon_{k}-\epsilon_{a}\right)\left(\epsilon_{j}-\epsilon_{a}\right)} \\
& +\sum_{i, a b c} \frac{f_{a i} f_{i b} \Delta v_{b c} \Delta v_{c a}}{\left(\epsilon_{i}-\epsilon_{a}\right)\left(\epsilon_{i}-\epsilon_{b}\right)\left(\epsilon_{i}-\epsilon_{c}\right)}-\sum_{i j, a b} \frac{f_{a i} \Delta v_{i j} f_{j b} \Delta v_{b a}}{\left(\epsilon_{i}-\epsilon_{a}\right)\left(\epsilon_{j}-\epsilon_{a}\right)\left(\epsilon_{j}-\epsilon_{b}\right)}-\sum_{i j, a b} \frac{f_{a i} \Delta v_{i j} f_{j b} \Delta v_{b a}}{\left(\epsilon_{i}-\epsilon_{a}\right)\left(\epsilon_{i}-\epsilon_{b}\right)\left(\epsilon_{j}-\epsilon_{b}\right)}+\ldots,
\end{aligned}
$$

where $\Delta v_{p q}=\left\langle\psi_{p}\left|\hat{f}-\hat{h}_{0}\right| \psi_{q}\right\rangle$, and $\Delta v_{i a}=f_{i a}=\left\langle\psi_{i}|\hat{f}| \psi_{a}\right\rangle$. To see how the infinite-order summation in Eq. (C6) is carried out, we rearrange the expression as follows:

$$
\begin{aligned}
E_{\mathrm{c}}^{\mathrm{rSE}}= & \sum_{i j, a b} \frac{f_{a i} \delta_{i j} \delta_{a b} f_{j b}}{\epsilon_{i}-\epsilon_{a}}+\sum_{i j, a b} \frac{f_{a i}\left(\Delta v_{a b} \delta_{i j}-\Delta v_{i j} \delta_{a b}\right) f_{j b}}{\left(\epsilon_{i}-\epsilon_{a}\right)\left(\epsilon_{j}-\epsilon_{b}\right)} \\
& +\sum_{i j k, a b c} \frac{f_{a i}\left(\Delta v_{i k} \Delta v_{k j} \delta_{a c} \delta_{b c}+\Delta v_{b c} \Delta v_{c a} \delta_{i k} \delta_{k j}-\Delta v_{i k} \Delta v_{b c} \delta_{j k} \delta_{a c}-\Delta v_{k j} \Delta v_{c a} \delta_{i k} \delta_{b c}\right) f_{j b}}{\left(\epsilon_{i}-\epsilon_{a}\right)\left(\epsilon_{k}-\epsilon_{c}\right)\left(\epsilon_{j}-\epsilon_{b}\right)}+\cdots \\
= & \sum_{i j, a b} \frac{f_{a i}}{\epsilon_{i}-\epsilon_{a}}\left[\delta_{i j} \delta_{a b}+\Omega_{i a, j b}+\left(\Omega^{2}\right)_{i a, j b}+\cdots\right] f_{j b}=\sum_{i j, a b} \frac{f_{a i}}{\epsilon_{i}-\epsilon_{a}}\left[(I-\Omega)^{-1}\right]_{i a, j b} f_{j b},
\end{aligned}
$$

where we have introduced the $\Omega$ matrix, defined as

$$
\Omega_{i a, j b}=\frac{\Delta v_{b a} \delta_{i j}-\Delta v_{i j} \delta_{a b}}{\epsilon_{j}-\epsilon_{b}} .
$$

Further denoting $A_{i a, j b}=\left(\epsilon_{i}-\epsilon_{a}\right) \delta_{i j} \delta_{a b}$, one observes

$$
\begin{aligned}
\frac{1}{\epsilon_{i}-\epsilon_{a}}\left[(I-\Omega)^{-1}\right]_{i a, j b} & =\left[A^{-1}(I-\Omega)^{-1}\right]_{i a, j b} \\
& =\left[(A-\Omega A)^{-1}\right]_{i a, j b}
\end{aligned}
$$

and

$$
\begin{aligned}
(A-\Omega A)_{i a, j b} & =\left(\epsilon_{i}-\epsilon_{a}\right) \delta_{i j} \delta_{a b}+\Delta v_{i j} \delta_{a b}-\Delta v_{b a} \delta_{i j} \\
& =f_{i j} \delta_{a b}-f_{a b} \delta_{i j},
\end{aligned}
$$

where $f_{i j}=\epsilon_{i} \delta_{i j}+\Delta v_{i j}, \quad f_{a b}=\epsilon_{a} \delta_{a b}+\Delta v_{a b}$ have been used. It follows that

$$
E_{\mathrm{c}}^{\mathrm{rSE}}=\sum_{i j, a b} f_{a i}\left[(A-\Omega A)^{-1}\right]_{i a, j b} f_{j b} .
$$

We observe that the rSE energy expressed in terms of canonical orbitals via Eqs. (C9) and (C10) has the same mathematical structure as the second-order SE energy expressed in terms of noncanonical orbitals given by Eqs. (C3) and (C4). The difference is that now the corresponding matrix elements in the denominator are evaluated using the Fock operator $\hat{f}$ instead of the KS Hamiltonian operator $\hat{h}^{0}$.
To simplify the evaluation of Eq. (C10), one can rotate the occupied orbitals and unoccupied orbitals separately, such that the Fock matrix becomes diagonal in the occupied and unoccupied subspaces. This procedure is called semicanonicalization. To be more precise, suppose there are transformation matrices $\mathcal{O}$ and $\mathcal{U}$ which diagonalize the $f_{i j}$ and $f_{a b}$ blocks separately:

$$
\sum_{k} f_{i k} \mathcal{O}_{k j}=\mathcal{O}_{i j} \tilde{\epsilon}_{j}, \quad \sum_{c} f_{a c} \mathcal{U}_{c b}=\mathcal{U}_{a b} \tilde{\epsilon}_{b} .
$$

We then have

$$
\sum_{k l, c d} \mathcal{O}_{i k}^{*} \mathcal{U}_{a c}^{*}(A-\Omega A)_{k c, l d} \mathcal{O}_{l j} \mathcal{U}_{d b}=\delta_{i j} \delta_{a b}\left(\tilde{\epsilon}_{j}-\tilde{\epsilon}_{b}\right)
$$

or, equivalently,

$$
\left[(A-\Omega A)^{-1}\right]_{i a, j b}=\sum_{k, c} \mathcal{O}_{i k} \mathcal{U}_{a c}\left(\tilde{\epsilon}_{k}-\tilde{\epsilon}_{c}\right)^{-1} \mathcal{O}_{k j}^{*} \mathcal{U}_{c b}^{*} .
$$

Inserting Eq. (C13) into (C10), one arrives at

$$
E_{\mathrm{c}}^{\mathrm{rSE}}=\sum_{i a} \frac{\tilde{f}_{a i} \tilde{f}_{i a}}{\tilde{\epsilon}_{i}-\tilde{\epsilon}_{a}},
$$

where

$$
\tilde{f_{i a}}=\sum_{j b} \mathcal{O}^{*}{ }_{i j} \mathcal{U}^{*}{ }_{a b} f_{j b}
$$


Thus, the final expression for $\mathrm{rSE}$ has the same form as that for SE, only the eigenvalues $\epsilon_{i}, \epsilon_{a}$ and the "transition amplitude" $f_{i a}$ have to be reinterpreted. The actual implementation following Eqs. (C11), (C14), and (C15) is straightforward.

\section{APPENDIX D: BASIS CONVERGENCE}

Figure 12 shows the convergence behavior of the rPT2 binding energy of the methane dimer (in its equilibrium geometry) with respect to the FHI-AIMS NAO "tier-N" basis as well as Dunning's "cc-pVXZ" and "aug-cc-pVXZ" basis. The methane dimer is dominated by the dispersion interaction, and the so-called "diffuse functions" are needed to accurately describe this interaction. The difference between the "ccpVXZ" and "aug-cc-pVXZ" results highlight the importance of including "diffuse functions." For the methane dimer, the "tier-N" series exhibits a faster convergence than "cc-pVXZ" but a slower convergence than "aug-cc-pVXZ" for BSSEcorrected binding energies. When adding diffuse functions from aug-cc-pV5Z to "tier 3/4" (called "t3/4+a5Z-d" in Fig. 12), results of similar quality as the full aug-cc-pV5Z basis are obtained.

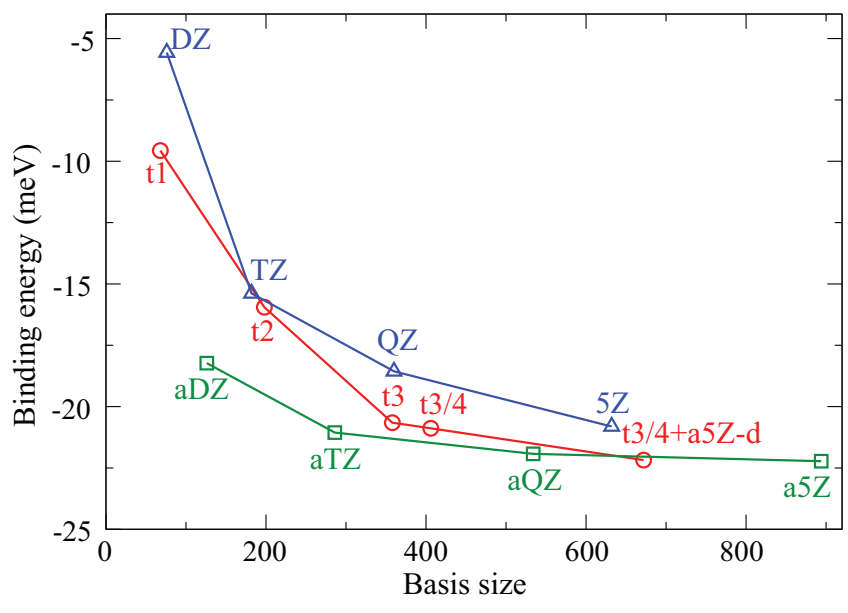

FIG. 12. (Color online) The rPT2@PBE binding energy of the methane dimer in its equilibrium geometry as a function of the basis set size. "XZ" and "aXZ" $(X=D, T, Q, 5)$ denote the Dunning "cc-pVXZ" and "aug-cc-pVXZ" basis respectively, whereas "tN" denotes the FHI-aims "tier $\mathrm{N}$ " basis. " $\mathrm{t} 3 / 4$ " here means tier 4 basis for $\mathrm{C}$ and tier 3 basis for $\mathrm{H}$ (note that a tier 4 basis for $\mathrm{H}$ is not available). " $\mathrm{t} 3 / 4+\mathrm{a} 5 \mathrm{Z}-\mathrm{d}$ " corresponds to the NAO "tier $3 / 4$ " plus diffuse functions from aug-cc-pV5Z. The BSSE is corrected.
${ }^{1}$ P. Hohenberg and W. Kohn, Phys. Rev. 136, B864 (1964).

${ }^{2}$ W. Kohn and L. J. Sham, Phys. Rev. 140, A1133 (1965).

${ }^{3}$ J. P. Perdew and K. Schmidt, in Density Functional Theory and its Application to Materials, edited by V. Van Doren, C. Van Alsenoy, and P. Geerlings (AIP, Melville, NY, 2001).

${ }^{4}$ D. Bohm and D. Pines, Phys. Rev. 92, 609 (1953).

${ }^{5}$ M. Gell-Mann and K. A. Brueckner, Phys. Rev. 106, 364 (1957).

${ }^{6}$ D. C. Langreth and J. P. Perdew, Phys. Rev. B 15, 2884 (1977).

${ }^{7}$ O. Gunnarsson and B. I. Lundqvist, Phys. Rev. B 13, 4274 (1976).

${ }^{8}$ A. Heßelmann and A. Görling, Mol. Phys. 109, 2473 (2011).

${ }^{9}$ H. Eshuis, J. E. Bates, and F. Furche, Theor. Chem. Acc. 131, 1084 (2012).

${ }^{10}$ X. Ren, P. Rinke, C. Joas, and M. Scheffler, J. Mater. Sci. 47, 7447 (2012).

${ }^{11}$ F. Furche, Phys. Rev. B 64, 195120 (2001).

${ }^{12}$ P. J. Feibelman, B. Hammer, J. K. Nørskov, F. Wagner, M. Scheffler, R. Stumpf, R. Watwe, and J. Dumestic, J. Phys. Chem. B 105, 4018 (2001).

${ }^{13}$ X. Ren, P. Rinke, and M. Scheffler, Phys. Rev. B 80, 045402 (2009).

${ }^{14}$ L. Schimka, J. Harl, A. Stroppa, A. Grüneis, M. Marsman, F. Mittendorfer, and G. Kresse, Nat. Mater. 9, 741 (2010).

${ }^{15}$ M. Casadei, X. Ren, P. Rinke, A. Rubio, and M. Scheffler, Phys. Rev. Lett. 109, 146402 (2012).

${ }^{16}$ J. Harl and G. Kresse, Phys. Rev. B 77, 045136 (2008).

${ }^{17}$ J. Harl and G. Kresse, Phys. Rev. Lett. 103, 056401 (2009).

${ }^{18}$ B. G. Janesko, T. M. Henderson, and G. E. Scuseria, J. Chem. Phys. 130, 081105 (2009).

${ }^{19}$ B. G. Janesko, T. M. Henderson, and G. E. Scuseria, J. Chem. Phys. 131, 034110 (2009).

${ }^{20}$ J. Toulouse, I. C. Gerber, G. Jansen, A. Savin, and J. G. Ángyán, Phys. Rev. Lett. 102, 096404 (2009).
${ }^{21}$ X. Ren, A. Tkatchenko, P. Rinke, and M. Scheffler, Phys. Rev. Lett. 106, 153003 (2011).

${ }^{22}$ D. Lu, Y. Li, D. Rocca, and G. Galli, Phys. Rev. Lett. 102, 206411 (2009).

${ }^{23}$ H. Eshuis and F. Furche, J. Phys. Chem. Lett. 2, 983 (2011).

${ }^{24}$ J. Paier, B. G. Janesko, T. M. Henderson, G. E. Scuseria, A. Grüneis, and G. Kresse, J. Chem. Phys. 132, 094103 (2010); 133, 179902(E) (2010).

${ }^{25}$ F. Caruso, D. R. Rohr, M. Hellgren, X. Ren, P. Rinke, A. Rubio, and M. Scheffler, Phys. Rev. Lett. 110, 146403 (2013).

${ }^{26}$ Z. Yan, J. P. Perdew, and S. Kurth, Phys. Rev. B 61, 16430 (2000).

${ }^{27}$ A. Grüneis, M. Marsman, J. Harl, L. Schimka, and G. Kresse, J. Chem. Phys. 131, 154115 (2009).

${ }^{28}$ A. Heßelmann, J. Chem. Phys. 134, 204107 (2011).

${ }^{29}$ A. Heßelmann and A. Görling, Phys. Rev. Lett. 106, 093001 (2011).

${ }^{30}$ A. Ruzsinszky, J. P. Perdew, and G. I. Csonka, J. Chem. Phys. 134, 114110 (2011)

${ }^{31}$ J. G. Ángyán, R.-F. Liu, J. Toulouse, and G. Jansen, J. Chem. Theory Comput. 7, 3116 (2011).

${ }^{32}$ T. Olsen and K. S. Thygesen, Phys. Rev. B 86, 081103(R) (2012).

${ }^{33}$ D. L. Freeman, Phys. Rev. B 15, 5512 (1977).

${ }^{34}$ J. Goldstone, Proc. R. Soc. London, Ser. A 239, 267 (1957).

${ }^{35}$ J. Paier, X. Ren, P. Rinke, G. E. Scuseria, A. Grüneis, G. Kresse, and M. Scheffler, New J. Phys. 14, 043002 (2012).

${ }^{36}$ P. Jurečka, J. Šponer, J. Černý, and P. Hobza, Phys. Chem. Chem. Phys. 8, 1985 (2006).

${ }^{37}$ J. Řezáč, P. Jurečka, K. E. Riley, J. Černý, H. Valdes, K. Pluháčková, K. Berka, T. Řezáč, M. Pitoňák, J. Vondrášek et al., Collect. Czech. Chem. Commun. 73, 1261 (2008).

${ }^{38}$ J. Řezáč, K. E. Riley, and P. Hobza, J. Chem. Theory Comput. 7, 2427 (2011). 
${ }^{39}$ L. A. Curtiss, K. Raghavachari, P. C. Redfern, and J. A. Pople, J. Chem. Phys. 106, 1063 (1997).

${ }^{40}$ Y. Zhao, N. González-García, and D. G. Truhlar, J. Phys. Chem. A 109, 2012 (2005).

${ }^{41}$ Y. Zhao and D. G. Truhlar, J. Chem. Phys. 125, 194101 (2006).

${ }^{42}$ G. Jansen, R.-F. Liu, and J. G. Ángyán, J. Chem. Phys. 133, 154106 (2010).

${ }^{43}$ V. Blum, F. Hanke, R. Gehrke, P. Havu, V. Havu, X. Ren, K. Reuter, and M. Scheffler, Comput. Phys. Commun. 180, 2175 (2009).

${ }^{44}$ X. Ren, P. Rinke, V. Blum, J. Wieferink, A. Tkatchenko, A. Sanfilippo, K. Reuter, and M. Scheffler, New J. Phys. 14, 053020 (2012).

${ }^{45}$ F. Furche, J. Chem. Phys. 129, 114105 (2008).

${ }^{46}$ G. E. Scuseria, T. M. Henderson, and D. C. Sorensen, J. Chem. Phys. 129, 231101 (2008).

${ }^{47}$ T. M. Henderson and G. E. Scuseria, Mol. Phys. 108, 2511 (2010).

${ }^{48}$ B. I. Dunlap, J. W. D. Connolly, and J. R. Sabin, J. Chem. Phys. 71, 3396 (1979).

${ }^{49}$ M. Feyereisen, G. Fitzgerald, and A. Komornicki, Chem. Phys. Lett. 208, 359 (1993).

${ }^{50}$ F. Weigend, M. Häser, H. Patzelt, and R. Ahlrichs, Chem. Phys. Lett. 294, 143 (1998).

${ }^{51}$ A. Görling and M. Levy, Phys. Rev. B 47, 13105 (1993).

${ }^{52}$ R. J. Bartlett, Mol. Phys. 108, 3299 (2010).

${ }^{53}$ H. Jiang and E. Engel, J. Chem. Phys. 125, 184108 (2006).

${ }^{54}$ R. J. Bartlett, I. Grabowski, S. Hirata, and S. Ivanov, J. Chem. Phys. 125, 204105 (2006).

${ }^{55}$ I. V. Schweigert, V. F. Lotrich, and R. J. Bartlett, J. Chem. Phys. 125, 104108 (2006).

${ }^{56}$ A. Szabo and N. S. Ostlund, Modern Quantum Chemistry: Introduction to Advanced Electronic Structure Theory (McGraw-Hill, New York, 1989).

${ }^{57}$ A. L. Fetter and J. D. Walecka, Quantum Theory of Many-Particle Systems (McGraw-Hill, New York, 1971).

${ }^{58}$ A. Grüneis, M. Marsman, and G. Kresse, J. Chem. Phys. 133, 074107 (2010).

${ }^{59} \mathrm{X}$. Ren et al. (unpublished).
${ }^{60}$ J. Harl, L. Schimka, and G. Kresse, Phys. Rev. B 81, 115126 (2010).

${ }^{61}$ L. A. Curtiss, P. C. Redfern, and K. Raghavachari, J. Chem. Phys. 123, 124107 (2005).

${ }^{62}$ A. Szabo and N. S. Ostlund, J. Chem. Phys. 67, 4351 (1977).

${ }^{63}$ J. F. Dobson, in Topics in Condensed Matter Physics, edited by M. P. Das (Nova, New York, 1994).

${ }^{64}$ J. F. Dobson and T. Gould, J. Phys.: Condens. Matter 24, 073201 (2012).

${ }^{65}$ D. Lu, H.-V. Nguyen, and G. Galli, J. Chem. Phys. 133, 154110 (2010).

${ }^{66}$ F. Furche and T. Van Voorhis, J. Chem. Phys. 122, 164106 (2005).

${ }^{67}$ Y. Li, D. Lu, H.-V. Nguyen, and G. Galli, J. Phys. Chem. A 114, 1944 (2010).

${ }^{68}$ W. Zhu, J. Toulouse, A. Savin, and J. G. Ángyán, J. Chem. Phys. 132, 244108 (2010).

${ }^{69}$ J. Toulouse, W. Zhu, A. Savin, G. Jansen, and J. G. Ángyán, J. Chem. Phys. 135, 084119 (2011).

${ }^{70}$ K. T. Tang and J. P. Toennies, J. Chem. Phys. 118, 4976 (2003).

${ }^{71}$ W. Klopper and J. Noga, J. Chem. Phys. 103, 6127 (1995).

${ }^{72}$ E. F. Laschuk, M. M. Martins, and S. Evangelisti, Int. J. Quantum Chem. 95, 303 (2003).

${ }^{73}$ J. T. H. Dunning, J. Chem. Phys. 90, 1007 (1989).

${ }^{74}$ S. F. Boys and F. Bernardi, Mol. Phys. 19, 553 (1970).

${ }^{75}$ T. Takatani, E. G. Hohenstein, M. Malagoli, M. S. Marshall, and C. D. Sherrill, J. Chem. Phys. 132, 144104 (2010).

${ }^{76}$ H. Eshuis and F. Furche, J. Chem. Phys. 136, 084105 (2012).

${ }^{77}$ E. Fabiano and F. Dalla Sala, Theor. Chem. Acc. 131, 1278 (2012).

${ }^{78}$ L. Gráfová, M. Pitoňák, J. Řezáč, and P. Hobza, J. Chem. Theory Comput. 6, 2365 (2010).

${ }^{79}$ D. Feller and K. A. Peterson, J. Chem. Phys. 108, 154 (1998).

${ }^{80}$ Q.-M. Hu, K. Reuter, and M. Scheffler, Phys. Rev. Lett. 98, 176103 (2007).

${ }^{81}$ E. Kaxiras, Atomic and Electronic Structure of Solids (Cambridge University Press, Cambridge, 2003).

${ }^{82} \mathrm{http} / / / \mathrm{cccbdb}$. nist.gov/.

${ }^{83}$ Gaussian Development Version, Revision G.01, M. J. Frisch et al., Gaussian, Inc., Wallingford CT, 2007.

${ }^{84}$ See, e.g., the Supplemental Material of Ref. 21 for a general proof. 